\title{
GABAergic Local Interneurons Shape Female Fruit Fly Response to Mating Songs
}

\author{
Daichi Yamada, Hiroshi Ishimoto, Xiaodong Li, Tsunehiko Kohashi, Yuki Ishikawa, and ๑Azusa Kamikouchi \\ Graduate School of Science, Nagoya University, Chikusa, Nagoya, Aichi 464-8602, Japan
}

\begin{abstract}
Many animals use acoustic signals to attract a potential mating partner. In fruit flies (Drosophila melanogaster), the courtship pulse song has a species-specific interpulse interval (IPI) that activates mating. Although a series of auditory neurons in the fly brain exhibit different tuning patterns to IPIs, it is unclear how the response of each neuron is tuned. Here, we studied the neural circuitry regulating the activity of antennal mechanosensory and motor center (AMMC)-B1 neurons, key secondary auditory neurons in the excitatory neural pathway that relay song information. By performing $\mathrm{Ca}^{2+}$ imaging in female flies, we found that the IPI selectivity observed in AMMC-B1 neurons differs from that of upstream auditory sensory neurons [Johnston's organ (JO)-B]. Selective knock-down of a GABA $\mathrm{A}_{\mathrm{A}}$ receptor subunit in AMMC-B1 neurons increased their response to short IPIs, suggesting that GABA suppresses AMMC-B1 activity at these IPIs. Connection mapping identified two GABAergic local interneurons that synapse with AMMC-B1 and JO-B. $\mathrm{Ca}^{2+}$ imaging combined with neuronal silencing revealed that these local interneurons, AMMC-LN and AMMC-B2, shape the response pattern of AMMC-B1 neurons at a $15 \mathrm{~ms}$ IPI. Neuronal silencing studies further suggested that both GABAergic local interneurons suppress the behavioral response to artificial pulse songs in flies, particularly those with a $15 \mathrm{~ms}$ IPI. Altogether, we identified a circuit containing two GABAergic local interneurons that affects the temporal tuning of AMMC-B1 neurons in the song relay pathway and the behavioral response to the courtship song. Our findings suggest that feedforward inhibitory pathways adjust the behavioral response to courtship pulse songs in female flies.
\end{abstract}

Key words: auditory neurons; courtship song; Drosophila melanogaster; feedforward inhibition; neural circuit

Significance Statement

To understand how the brain detects time intervals between sound elements, we studied the neural pathway that relays speciesspecific courtship song information in female Drosophila melanogaster. We demonstrate that the signal transmission from auditory sensory neurons to key secondary auditory neurons antennal mechanosensory and motor center (AMMC)-B1 is the first-step to generate time interval selectivity of neurons in the song relay pathway. Two GABAergic local interneurons are suggested to shape the interval selectivity of AMMC-B1 neurons by receiving auditory inputs and in turn providing feedforward inhibition onto AMMC-B1 neurons. Furthermore, these GABAergic local interneurons suppress the song response behavior in an intervaldependent manner. Our results provide new insights into the neural circuit basis to adjust neuronal and behavioral responses to a species-specific communication sound.

\section{Introduction}

Many animals use sounds as sexual and social signals for communication (Bradbury and Vehrencamp, 2011). Among the various

\footnotetext{
Received Dec. 26, 2017; revised March 19, 2018; accepted March 26, 2018

Author contributions: A.K. designed research; D.Y., X.L., and A.K. performed research; D.Y., H.I., T.K., Y.I., and A.K. analyzed data; D.Y., H.I., T.K., Y.I., and A.K. wrote the paper.

This work was supported by the Ministry of Education, Culture, Sports, Science, and Technology, Japan (Grantin-Aid for Scientific Research B $16 H 04655$ to A.K., Grants-in-Aid for Scientific Research on Innovate Area "Memory Dynamism" 25115007 to A.K., Challenging Research Exploratory Grant 17K19450 to A.K., Grant-in-Aid for Scientific Research C 15K07147 to H.I., Grant-in-Aid for Young Scientists B 26870264 to Y.I., and Grant-in-Aid for Japan Society for the Promotion of Science Research Fellow 15J04760 to D.Y.) and by the Inamori Foundation, Japan (Research Grant to H.I.). We thank Dr. Yoichi Oda, Dr. Eriko Matsuo, and Dr. Nao Morimoto for discussions; Miki Kuno, Yumi Maki, and Yuki Ishikawa for fly maintenance; Tomonori Asai for illustrations; and the Bloomington Drosophila Stock Center (BDSC), Vienna Drosophila Resource Center (VDRC), and Developmental Studies Hybridoma Bank for reagents.

The authors declare no competing financial interests.
}

acoustic features that comprise communication sounds, the time interval between acoustic elements typically is important for discriminating conspecific sounds from heterospecific sounds, particularly in lower vertebrates and insects (Pollack, 2001). Auditory neural circuits in the CNS process the intervals of conspecific sounds (Rose, 2014; Hedwig, 2016) and various animals such as frogs and crickets have a series of neurons that respond to specific intervals (Alexander, 1962; Rose and Capranica, 1983; Schöneich et al., 2015). In frogs, some midbrain neurons respond to a specific pulse rate (Alder and Rose, 1998, 2000; Edwards et al.,

Correspondence should be addressed to Azusa Kamikouchi, Division of Biological Science, Graduate School of Science, Nagoya University, Chikusa, Nagoya, Aichi, 464-8602, Japan. E-mail: kamikouchi@bio.nagoya-u.ac.jp. DOI:10.1523/JNEUROSCI.3644-17.2018

Copyright $\odot 2018$ the authors $\quad 0270-6474 / 18 / 384329-19 \$ 15.00 / 0$ 
2007; Rose et al., 2011). Female crickets respond to male cricket's songs that have a specific interpulse interval (IPI) and investigators have identified auditory interneurons that respond selectively to a specific song (Kostarakos and Hedwig, 2012; Schöneich et al., 2015). In frogs and crickets, excitatory and inhibitory inputs are suggested to interact to generate IPI selectivity (Rose, 2014; Hedwig, 2016). These findings suggest that excitatory-inhibitory combinations are important for generating the interval selectivity of these neurons. A direct causality between the role of the inhibitory system in the circuit and the resulting network output or behavior remains elusive, however, mainly due to the lack of molecular genetic tools to manipulate individual neurons.

In fruit flies (Drosophila melanogaster), acoustic signals generated by a courting male, a so-called the courtship song, are important for species recognition during courtship behavior. The courtship song comprises a sine song and a pulse song (von Schilcher, 1976); the pulse song has a species-specific IPI ( $35 \mathrm{~ms}$ in D. melanogaster) that strongly promotes copulation in paired flies (Kyriacou and Hall, 1982; Ritchie et al., 1999). The antennal ear of fruit flies, Johnston's organ (JO), houses cholinergic mechanosensory neurons called JO neurons (Ishikawa et al., 2017) that project to the antennal mechanosensory and motor center (AMMC) in the brain (see Fig. 1A) (Kamikouchi et al., 2006). Recent studies identified the auditory neural pathway in the brains of males and females that is responsible for detecting temporal patterns in the courtship song. This pathway comprises a subset of JO neurons (subgroup-B neurons; JO-B), second-order AMMC-B1 (also known as aPN1) neurons, and sexually dimorphic downstream neurons (Lai et al., 2012; Vaughan et al., 2014; Zhou et al., 2014, 2015). In females, AMMC-B1 neurons have a band-pass response to the IPI and have a peak $\mathrm{Ca}^{2+}$ response at $25 \mathrm{~ms}$ IPI (Vaughan et al., 2014). These neurons are necessary for enhancing female receptivity for copulation (Vaughan et al., 2014) and are regarded as important relay neurons that transmit courtship song information to $\mathrm{pC} 1$ neurons, which respond to $35 \mathrm{~ms}$ IPI pulse songs and regulate female receptivity to a courting male (Zhou et al., 2014). Males also have an auditory pathway for perceiving pulse songs that includes JO-B, AMMC-B1, vPN1, and pC1 neurons. Neurons in this pathway tune the perception of IPIs by multistage transformations. These transformations eventually lead to the close matching between the response of $\mathrm{pC} 1$ neurons, which includes the courtship command-like neurons $\mathrm{P} 1$ in males, and the behavioral response to songs with different IPIs (Zhou et al., 2015). Together, these results suggest that the IPI selectivity of the fly's auditory system is sequentially transformed from JO-B neurons to $\mathrm{pC} 1$ neurons in both males and females. The IPI selectivity of AMMC-B1 neurons is an important step in the transformation, but how such selectivity emerges remains unknown.

Here, we unravel the neuronal mechanism that shapes the IPI selectivity of AMMC-B1 neurons in females. In this mechanism, two inhibitory GABAergic local interneurons form circuits in the JO-B to AMMC-B1 pathway and shape the response pattern of AMMC-B1 neurons. Furthermore, these GABAergic local interneurons suppress the song response behavior, particularly at a $15 \mathrm{~ms}$ IPI. These findings provide insight into the neural basis for information processing of the species-specific courtship song.

\section{Materials and Methods}

Experimental animals. Fruit flies were raised on standard yeast-based media at $25^{\circ} \mathrm{C}$ and $40-60 \%$ relative humidity unless otherwise noted. Canton-S flies were used as a wild-type. The following fly strains were used for the GAL4/UAS (Brand and Perrimon, 1993) and LexA/lexAop (Lai and Lee, 2006; Pfeiffer et al., 2010) technique: R45D07-GAL4 (RRID:
BDSC_49562), R55C02-GAL4 (RRID:BDSC_39104), R26C02-GAL4 (Matsuo et al., 2016), Rdl-GAL4 (RRID:BDSC_66509), and R45D07LexA (RRID:BDSC_54103) from Bloomington stock center (Bloomington, IN); VT022100-GAL4 (RRID:FlyBase_FBst0485354), VT021793-GAL4 (RRID:FlyBase_FBst0485265), and VT017247-GAL4 (RRID:FlyBase_FBst0484682) from VDRC stock center (Vienna, Austria); F-GAL4 (Kim et al., 2003) (courtesy of Dr. Changsoo Kim); NV0116-LexA and lexAop-rCD2:: GFP (Matsuo et al., 2016) (courtesy of Dr. Kei Ito); UAS-GCaMP6m (RRID:BDSC_42750), UAS-GCaMP6f (RRID:BDSC_42747), LexAopGCaMP6f(RRID:BDSC_44277), UAS-IVS-mCD8::RFP(RRID:BDSC_32219), UAS-IVS-mCD8::GFP (RRID:BDSC_32186), UAS-D $27:: G F P$ (RRID: BDSC_39692), UAS-TNT (RRID:BDSC_28838 and RRID:BDSC_28997), UAS-IMPTNT (RRID:BDSC_28840 and RRID:BDSC_28841), and UAS-RdlRNAi (RRID:BDSC_52903) from Bloomington Stock Center (Bloomington, IN); LexAop-CD4::GFP11 and UAS-CD4::spGFP1-10 (courtesy of Dr. Kristin Scott); UAS-GFP S65T (T2 strain, courtesy of Dr. Barry Dickson); UASneuronal synaptobrevin::GFP ( $n S y b:: G F P$ ) combined with UAS-DsRed S197Y (courtesy of Dr. Kei Ito). To overlay AMMC-B1 neurons and $R d l-G A L 4$ neurons by using registration technique, we used $R 45 D 07-$ LexA and Rdl-GAL4 drivers (see Fig. 2A, right). R45D07-LexA driver is a LexA counterpart of $R 45 D 07-G A L 4$ that was used in the $\mathrm{Ca}^{2+}$ imaging and the female receptivity of AMMC-B1 neurons. We confirmed that R45D07GAL4 and R45D07-LexA both labeled AMMC-B1 neurons (Fig. 1-1, available at https://doi.org/10.1523/JNEUROSCI.3644-17.2018.f1-1, Fig. 3-1, available at https://doi.org/10.1523/JNEUROSCI.3644-17.2018.f3-1). Fly genotypes are shown in Table 1 .

$\mathrm{Ca}^{2+}$ imaging data acquisition. We used GAL4 and LexA driver lines to express the $\mathrm{Ca}^{2+}$ indicator GCaMP6f (see Table 1 for fly genotypes) (Chen et al., 2013). We confirmed that these driver lines labeled the target neurons specifically, at least in the region of interest (ROI) in which we measured the GCaMP6f fluorescence. $\mathrm{Ca}^{2+}$ imaging was performed in female flies as described previously with minor modifications (Matsuo et al., 2014). Flies were anesthetized on ice for $<1 \mathrm{~min}$ and affixed onto an imaging plate using silicon grease (SH 44M; Toray) with the ventral side up. The mouthpart of the fly was removed using fine tweezers to open a window to monitor brain fluorescence. A drop of an adult hemolymphlike saline solution containing the following (in mM): $108 \mathrm{NaCl}, 5 \mathrm{KCl}, 2$ $\mathrm{CaCl}_{2}, 8.2 \mathrm{MgCl}_{2}, 4 \mathrm{NaHCO}_{3}, 1 \mathrm{NaH}_{2} \mathrm{PO}_{4}, 5$ trehalose, 10 sucrose, and 5 mM HEPES, pH 7.5 adjusted with $1 \mathrm{~m} \mathrm{NaOH}, 265$ mOsm, (Wang et al., 2003) was immediately added to prevent dehydration. A fluorescent microscope (Axio Imager.A2; Carl Zeiss) equipped with a water-immersion $20 \times$ objective lens [W Achroplan/W N-Achroplan, numerical aperture $(\mathrm{NA})=0.5$; Carl Zeiss], a spinning disc confocal head CSU-W1 (Yokogawa), and an OBIS 488 LS laser (Coherent Technologies) for excitation at $488 \mathrm{~nm}$ was used. To provide sound stimuli, a loudspeaker (Voice AR-10N; Daito) was located $\sim 11 \mathrm{~cm}$ from the antenna of the fly. Synthetic song stimuli, which comprised 20 pulse trains with different IPIs (15-105 ms) with a $167 \mathrm{~Hz}$ intrapulse frequency were delivered from the loudspeaker (Fig. 1B). Mean baseline-to-peak amplitude of its particle velocity was $24.8 \mathrm{~mm} / \mathrm{s}$. The fluorescent images were captured at 10 frames/s with an exposure time of $100 \mathrm{~ms}$ using an EM-CCD camera (ImagEM, C9100-13; Hamamatsu Photonics) at a resolution of $512 \times$ 512 pixels in water-cooled mode. In each individual, we measured the $\mathrm{Ca}^{2+}$ response to a series of acoustic stimuli for 3 trials with at least a $10 \mathrm{~s}$ intertrial interval between each acoustic stimulus. For imaging of flies expressing tetanus toxin (TNT), we used the R45D07-LexA driver to express GCaMP6f in AMMC-B1 neurons. AMMC-B1 neurons labeled by R45D07-LexA and R45D07-GAL4 drivers have similar response profiles to varying IPIs (Fig. $1 D$; Fig. 5-1 $A$, available at https://doi.org/10. 1523/JNEUROSCI.3644-17.2018.f5-1; Table 3; and Table 3-1, available at https://doi.org/10.1523/JNEUROSCI.3644-17.2018.t3-1). We confirmed the TNT and IMPTNT expressions in the target neurons by immunolabeling the brains with anti-TNT antibody after each imaging experiment (data not shown).

$\mathrm{Ca}^{2+}$ imaging data analysis. Imaging data were analyzed offline with ImageJ (version 1.47v; RRID:SCR_003070), Excel (Microsoft), and R (version 3.3.3, https://www.r-project.org; RRID:SCR_001905) software. Aberrations of serial images due to animal movement were aligned using an ImageJ plug-in TurboReg (http://bigwww.epfl.ch/thevenaz/turboreg/; 
RRID:SCR_014308). We set the ROI for each neuronal type as follows. For JO-B neurons, we set the ROI at the posterior region of JO neuron axons within the AMMC zone B. This region houses JO-B neuron output sites, which were detected in a previous study (Kamikouchi et al., 2006) by expressing the presynaptic marker GFP-tagged neuronal synaptobrevin (nSyb::GFP) (Estes et al., 2000). AMMC-B1 neurons innervate the ipsilateral AMMC zone B and the wedge (WED) on both sides of the brain (Matsuo et al., 2016) and have postsynaptic structures in the AMMC zone B and presynaptic structures in the WED (Lai et al., 2012; Vaughan et al., 2014; Matsuo et al., 2016); therefore, the postsynaptic (dendritic) and the presynaptic regions are located in distinct locations along the AMMC-B1 neurite. We therefore set the ROI in the dendritic region of $\mathrm{AMMC}-\mathrm{B} 1$ neurons within the $\mathrm{AMMC}$, which was detected by expressing the postsynaptic marker Dscam::GFP (Wang et al., 2004; Lai et al., 2012). For AMMC-LN and AMMC-B2 neurons, we set the ROI in their neurites in the AMMC, where both of presynaptic and postsynaptic sites are intermingled (Matsuo et al., 2016).

To assess the $\mathrm{Ca}^{2+}$ response, we calculated the relative fluorescence change $(\Delta F / F)$ of GCaMP6f as follows: $\Delta F / F=\left(F_{\mathrm{n}}-F_{\text {Base }}\right) / F_{\text {Base }}$, where the mean fluorescence intensity within an ROI at each time point $n\left(F_{\mathrm{n}}\right)$ was divided by the mean fluorescence intensity of the $\mathrm{Ca}^{2+}$ response during the $0.9 \mathrm{~s}$ preceding the stimulus onset $\left(F_{\text {Base }}\right)$. To evaluate the response to varying IPIs, we calculated the $\mathrm{Ca}^{2+}$ response obtained at each IPI stimulus in two different ways. One was the peak $\mathrm{Ca}^{2+}$ response, which represents the peak $\Delta F / F$ during stimulation and has been measured in previous studies of the fly auditory system (Vaughan et al., 2014; Zhou et al., 2015). The other was the integrated $\mathrm{Ca}^{2+}$ response, which represents the time integral of stimulation-evoked $\Delta F / F$. Peak $\mathrm{Ca}^{2+}$ response reflects maximum intracellular $\mathrm{Ca}^{2+}$ concentration $\left(\left[\mathrm{Ca}^{2+}\right]_{\mathrm{i}}\right)$ within an ROI (presynaptic and/or postsynaptic region) and thus indicates maximum instantaneous synaptic activity achieved during a stimulus train. A drawback of the peak $\mathrm{Ca}^{2+}$ response particularly in this study is that it is difficult to evaluate temporal processing, or IPIdependent modification of synaptic activity, by the auditory circuit. Even if there is no IPI-dependent modification of a single-pulse $\mathrm{Ca}^{2+}$ response during a stimulus train, the slow time course of the $\mathrm{Ca}^{2+}$ response can cause strong summation of $\left[\mathrm{Ca}^{2+}\right]_{i}$ at short IPIs. The summation, however, will be less significant at long IPIs. To circumvent such systematic problems and to detect IPI-dependent modification of $\mathrm{Ca}^{2+}$ flux evoked by each stimulus pulse, we introduced the integrated $\mathrm{Ca}^{2+}$ response. If individual stimulus pulses equally activate $\mathrm{Ca}^{2+}$ flux regardless of the IPI (namely a single pulse response is not tuned to the IPI) and result in an accumulation of GCaMP6f signals, then the integrated response would be constant across IPIs. We confirmed that the IPI responses that we observed in this study were within the dynamic range of GCaMP6f; in each neuronal type, pure tone stimulation ( 40 or $200 \mathrm{~Hz}$ for $1 \mathrm{~s}$ given at the beginning and the end of each measurement) evoked stronger peak response than the pulse trains of any IPIs. This result validates that the GCaMP6f responses to any IPIs in our measurement were not saturated. In the integrated $\mathrm{Ca}^{2+}$ response, we defined the response onset as the time of the stimulus onset and the end of the response as the time point when the $\Delta F / F$ amplitude returned to $10 \%$ of the maximum. For this calculation, we excluded the trial data in which $\Delta F / F$ did not return to baseline (between the values 0.05 and -0.05 ) at $1.9 \mathrm{~s}$ after the stimulus ended because a large shift in the resting fluorescence typically occurs when there is a relatively large drift in the focal plane. The integrated $\mathrm{Ca}^{2+}$ response therefore represents net $\mathrm{Ca}^{2+}$ flux elicited by 20 stimulus pulses, indicating integrated synaptic activity evoked by pulse sound. It is noteworthy that this parameter is a close approximation of the "total number of spikes elicited by a fixed number of stimuli," which is conventionally reported in electrophysiological studies on neural computation of IPI selectivity (Yan and Suga, 1996). To compare IPI selectivity among neurons or experiments, we also normalized the $\mathrm{Ca}^{2+}$ responses by the response to $105 \mathrm{~ms}$ IPI, which we defined as the reference response to compensate for response fluctuation across animals. To compare the response intensity between groups, we used the $\mathrm{Ca}^{2+}$ response without normalization, called the raw $\Delta F / F$ response. The resulting time trace data for each sound stimulus are shown as the mean value and SEM derived from $6-15$ flies.
Immunohistochemistry. Immunolabeling was performed in female flies unless otherwise noted, as described previously with minor modifications (Matsuo et al., 2016). Briefly, brains were fixed with $4 \%$ paraformaldehyde in PBS for 60-90 min on ice and subjected to the antibody labeling. Primary antibodies used in this study were as follows: rabbit anti-DsRed (Living Colors DsRed Polyclonal Antibody, Clontech, \#632496; RRID:AB_10013483; 1:1000 dilution) was used for detecting the mCD8:: RFP and DsRed. Mouse anti-GFP monoclonal (Sigma-Aldrich, \#G6539; RRID:AB_259941; 1:100 or 1:200 dilution) was used for detecting the reconstructed GFP (i.e., GFP reconstitution across synaptic partners, GRASP) signals. Chicken anti-GFP polyclonal (Abcam, \#13970; RRID: AB_300798; 1:5000 dilution) was used for detecting the CD4::spGFP110. Rabbit anti-GFP polyclonal (Invitrogen, \#A11122; RRID:AB_221569; 1:500, 1:1000 or 1:2000 dilution) was used for detecting the CD4:: spGFP1-10, mCD8::GFP, GFP S65T, GCaMP6m, and GCaMP6f. Rat anti-GFP monoclonal (Nacalai Tesque, \#04404-26; 1:500 or 1:1000 dilution) was used for detecting the GCaMP6f, nSyb::GFP, rCD2::GFP, and D $\alpha 7:: G F P$. Mouse anti-Bruchpilot nc82 (Developmental Studies Hybridoma Bank, \#nc82; RRID:AB_2314866; 1:20 dilution) was used to visualize neuropils in the brain. Rabbit anti-CD4 polyclonal (SigmaAldrich, HPA004252; RRID:AB_1078466; 1:500 dilution) was used for detecting the CD4::spGFP1-10 and CD4::spGFP11. Rabbit anti-tetanustoxin (Statens Serum Institut, \#POL 016; 1:2500 or 1:5000 dilution) was used to detect TNT expression.

Secondary antibodies used in this study were as follows: Alexa Fluor 488-conjugated anti-rat IgG (Jackson ImmunoResearch, \#112-545-167; RRID:AB_2338362; 1:300 dilution), Alexa Fluor 488-conjugated antirabbit IgG (Thermo Fisher Scientific, \#A11034; RRID:AB_2576217; 1:150 or 1:300 dilution), Alexa Fluor 488-conjugated anti-mouse IgG (Invitrogen, \#A11029; RRID:AB_138404; 1:300 dilution), Alexa Fluor 555-conjugated anti-mouse IgG (Invitrogen, \#A21424; RRID:AB_141780; 1:300 dilution), Alexa Fluor 555-conjugated anti-rabbit IgG (Invitrogen, \#A21429, RRID:AB_141761; 1:300 dilution), Alexa Fluor 647-conjugated anti-mouse IgG (Invitrogen, \#A21236; RRID:AB_141725; 1:300 dilution), Alexa Fluor 647-conjugated anti-chicken IgY (Thermo Fisher Scientific, \#A21449; RRID:AB_2535866; 1:300 dilution), and Alexa Fluor 647-conjugated anti-rabbit IgG (Thermo Fisher Scientific, \#A21245; RRID:AB_2535813; 1:300 dilution). Alexa Fluor 647 phalloidin (Thermo Fisher Scientific, \#A22287; RRID:AB_2620155; 1:50 dilution) was used to visualize F-actin.

Confocal microscopy and image processing. Serial optical sections of brains were obtained at $0.84 \mu \mathrm{m}$ intervals with a resolution of $512 \times 512$, $640 \times 640$, or $1024 \times 1024$ pixels by using an FV-1000D laser-scanning confocal microscope (Olympus) equipped with a silicone-oil-immersion $30 \times$ Plan-Apochromat objective lens ( $\mathrm{NA}=1.05$; Olympus). Confocal image datasets of R45D07-LexA neurons and Rdl-GAL4 neurons labeled with $\mathrm{rCD} 2:: \mathrm{GFP}$ and GFP, respectively, were digitally aligned to a template brain with nonrigid registration using the Computational Morphometry Toolkit (CMTK; RRID:SCR_002234) (Jefferis et al., 2007) (Fig. $2 A$, right). The single neuron images of AMMC-B1, AMMC-LN, and AMMC-B2 were modified from Matsuo et al. (2016) (see Fig. 3B). For the synaptic connections and morphology analysis, single or several sections of confocal datasets were merged using ImageJ. To identify AMMC zones B and D, we used the labeling pattern with nc82 antibody (Kamikouchi et al., 2009; Matsuo et al., 2014). To map the output sites of local interneurons (AMMC-LN and AMMC-B2) and the dendritic region of the AMMC-B1 neurons in the same brain, confocal image datasets of presynaptic sites labeled with nSyb::GFP and the dendritic region labeled with GFP were digitally aligned to a template brain using CMTK (RRID:SCR_002234) (Jefferis et al., 2007) (Fig. 3E). The size, contrast, and brightness of the images were adjusted using Illustrator CS6 (Adobe; RRID:SCR_014198) and ImageJ.

GRASP. GRASP was performed to detect membrane contacts between two neuronal types in female flies expressing the CD4::spGFP1-10 fragment in one neuronal type and the CD4::spGFP11 fragment in the other neuronal type using the GAL4/UAS and LexA/lexAop systems, respectively (Gordon and Scott, 2009). GRASP signals between AMMC-B1 neurons and each type of GABAergic local interneurons were analyzed in flies expressing CD4::spGFP11 in AMMC-B1 neurons and CD4::spGFP1-10 in 
each of the GABAergic local interneurons (AMMC-LN and AMMC-B2). To express CD4::spGFP11 in AMMC-B1 neurons, we used R45D07-LexA driver, a LexA counterpart of R45D07-GAL4. To visualize the reconstituted GFP in the GRASP analysis, mouse monoclonal anti-GFP antibody was used (Gordon and Scott, 2009). To detect CD4::spGFP1-10, we used rabbit polyclonal anti-GFP or chicken polyclonal anti-GFP antibodies as described previously (Gordon and Scott, 2009; Macpherson et al., 2015). To visualize the morphologies of AMMC-B1 and each GABAergic local interneuron simultaneously, we used rabbit polyclonal anti-CD4 antibody, which detects both CD4::spGFP fragments (Miyazaki et al., 2015). GRASP signals between JO neurons and GABAergic local interneurons (AMMC-LN and AMMC-B2) were analyzed in flies expressing CD4:: spGFP11 in JO neurons and CD4::spGFP1-10 in either AMMC-LN or AMMC-B2. To validate the specificity of the GRASP signals, we analyzed flies expressing CD4::spGFP1-10 in each GABAergic local interneuron, but not CD4::spGFP11 in JO neurons (Fig. 3-2, available at https://doi. org/10.1523/JNEUROSCI.3644-17.2018.f3-2). In this condition, although CD4::spGFP1-10 signals were detected in both GABAergic local interneurons, no GRASP signals were detected. For antibody information, see the "Immunohistochemistry" section in the Materials and Methods. For fly genotypes, see Table 1. For labeling patterns of each driver line, see Figure 3-1 (available at https://doi.org/10.1523/ JNEUROSCI.3644-17.2018.f3-1).

Female copulation assay. Female copulation assay was performed as described previously with minor modifications (Li et al., 2018). Wildtype males were used throughout the experiments to serve as the courtship partner for female flies. The courtship receptivity of wild-type and genetically manipulated females (see Table 1 for fly genotypes) was measured upon playback of the artificial pulse song. Flies of both sexes were collected within $8 \mathrm{~h}$ after eclosion. To prevent the males from emitting their own courtship song during the copulation assay, their wings were removed under ice anesthesia on the day of eclosion. The wings of the females remained intact. The flies were then kept in single-sex groups (up to 12 flies per vial) under a $12 \mathrm{~h}$ light/dark cycle until the experiments. The copulation assays were performed at $25 \pm 1^{\circ} \mathrm{C}$ and $50 \pm 10 \%$ relative humidity and within $4 \mathrm{~h}$ after light onset. A pair of male and female flies was placed into each circle-shaped courtship chamber $(15 \mathrm{~mm}$ diameter and $3 \mathrm{~mm}$ deep) with a plastic lid and meshed bottom by gentle aspiration without anesthesia. For the acoustic stimulus, we generated artificial pulse songs using the software Audacity (The Audacity Team, version 2.0; RRID:SCR_007198). These songs comprised the repetition of $1 \mathrm{~s}$ pulse burst and a subsequent $2 \mathrm{~s}$ pause (see Fig. $6 \mathrm{~A}$, bottom). The pulses in each burst had $167 \mathrm{~Hz}$ intrapulse frequency and three different IPIs $(15,25$, and $35 \mathrm{~ms})$. The duration of a single pulse in the pulse burst was $\sim 6 \mathrm{~ms}$. Mean baseline-to-peak amplitude of its particle velocity was 9.2 $\mathrm{mm} / \mathrm{s}$. The behavioral chamber was located above a loudspeaker (Daito Voice AR-10N, Japan) at a distance of $39 \mathrm{~mm}$. The sound playback started at the onset of the video recording and continued until the end of the recording. Digital video was recorded at 1 frame/s with a web camera (Logicool HD Webcam C270). Behavioral response of females to the pulse songs was evaluated by observing the copulation latency (time to mating) of a female paired with a wing-clipped wild-type male in the courtship chamber. Here, copulation was defined by observing the specific features as follows: (1) females permit a male to mount them for $>1$ min, (2) females reduce their locomotor activity with a mounting partner, and (3) females part their wings during the mounting (Manning, 1967). The accumulated copulation rate (CR) was calculated by the number of the successful copulations during the $30 \mathrm{~min}$ observation period. To estimate the suppression of the CR by each GABAergic local interneuron, we calculated the suppression index as follows:

$$
\text { suppression index }=1-\mathrm{CR}_{\mathrm{IMPTNT}} / \mathrm{CR}_{\mathrm{TNT}}
$$

where $\mathrm{CR}_{\mathrm{TNT}}$ and $\mathrm{CR}_{\mathrm{IMPTNT}}$ represented the $\mathrm{CR}$ at $30 \mathrm{~min}$ (gray shaded bars in Fig. $6 C, D$ ) in flies expressing TNT and its inactive form (IMPTNT), respectively.

Experimental design and statistical analysis. For immunolabeling and $\mathrm{Ca}^{2+}$ imaging of flies carrying R45D07-LexA as a driver, the flies were incubated at $29^{\circ} \mathrm{C}$ for $7-15 \mathrm{~d}$ after eclosion to enhance the expression level. For other cases, the flies were incubated at $25^{\circ} \mathrm{C}$ for the indicated

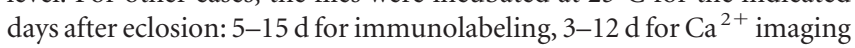
of GAL4 > GCaMP $6 f$ flies, 3-7 d for the copulation assay to $15 \mathrm{~ms}$ IPI song in TNT or IMPTNT expressing flies, and 5-10 d for other copulation assays.

For statistical analyses of $\mathrm{Ca}^{2+}$ imaging, we used the raw $\Delta F / F$ values. To compare the response patterns to varying IPIs between neuronal types (JO-B and AMMC-B1 in Fig. 1) or between conditions (Rdl knock-down and control AMMC-B1 neurons in Fig. 2C, TNT and IMPTNT flies in Fig. $5 B-D$ ), we applied two-way ANOVA with repeated measures because the dataset showed both normality and homogeneity of variance (Kolmogorov-Smirnov test and Bartlett test, respectively) (see Table 2). To compare the raw $\Delta F / F$ responses to each IPI between different conditions (see Rdl knock-down and control flies in Fig. 2B; TNT and IMPTNT flies in Fig. 5-1 B, available at https://doi.org/10.1523/JNEUROSCI.3644-17.2018.f5-1; Table 2, Table 3-1, available at https://doi.org/ 10.1523/JNEUROSCI.3644-17.2018.t3-1), the Mann-Whitney $U$ test with Bonferroni's correction for multiple comparisons was applied. To compare the $\mathrm{Ca}^{2+}$ responses to a series of IPIs in each GAL4 strain or condition (Figs. 1, 2C, 4, and 5B-D; Fig. 5-1A, available at https://doi.org/ 10.1523/JNEUROSCI.3644-17.2018.f5-1; Table 3; Table 3-1, available at https://doi.org/10.1523/JNEUROSCI.3644-17.2018.t3-1), one-way ANOVA with repeated measures followed by Bonferroni's correction for multiple comparisons was applied because each dataset showed normality and homogeneity of variance (Kolmogorov-Smirnov test and Bartlett test, respectively). To compare the copulation assay datasets (Fig. $6 B-D$; Fig. 6-2, available at https://doi.org/10.1523/JNEUROSCI.364417.2018.f6-2; Table 4; Table 4-1, available at https://doi.org/10.1523/ JNEUROSCI.3644-17.2018.t4-1), CR curves, which represent the accumulated CRs over time, were estimated by the Kaplan-Meier method and compared by log-rank test with the Cochran-Mantel-Haenszel method. The $p$-value was corrected using the Benjamini and Hochberg method to keep the false discovery rate $<0.05$ (Benjamini and Hochberg, 1995). The statistical analyses were conducted using R software (version 3.3.3) and Excel software (Microsoft) with dedicated software (Ekuseru-Toukei, 2012; Social Survey Research Information). Full details of statistical tests and scores are shown in Tables 2, 3, and 4; Table 3-1 (available at https://doi.org/10.1523/JNEUROSCI.3644-17.2018.t31); and Table 4-1 (available at https://doi.org/10.1523/JNEUROSCI.364417.2018.t4-1).

\section{Results}

\section{Transformation of song responses between JO-B and AMMC-B1}

To compare the song response properties of AMMC-B1 neurons and their upstream neurons in females, we performed in vivo $\mathrm{Ca}^{2+}$ imaging of JO-B and AMMC-B1 neurons (Fig. $1 B$ ). We expressed a $\mathrm{Ca}^{2+}$ sensor, GCaMP6f, in each neuronal type to monitor the neuronal activities during the artificial pulse songs of varying IPIs (see Table 1 for genotypes and Fig. 1-1, available at https://doi.org/10.1523/JNEUROSCI.3644-17.2018.f1-1, for the labeling patterns of each driver line; Kim et al., 2003; Matsuo et al., 2014, 2016; Vaughan et al., 2014).

To compare the IPI selectivity between JO-B and AMMC-B1 neurons at their transmission sites, we monitored the activity of JO-B neurons in their axon terminals where the output synapses are located (Kamikouchi et al., 2006) and in the dendritic region of AMMC-B1 neurons (Lai et al., 2012; Vaughan et al., 2014). Both JO-B and AMMC-B1 neurons responded to all artificial pulse songs tested (Fig. $1 C, D$ ). The pattern of integrated $\mathrm{Ca}^{2+}$ responses to different IPIs was statistically different between JO-B and AMMC-B1 (Table 2). In JO-B neurons, the integrated $\mathrm{Ca}^{2+}$ response was similar among all IPIs tested (Fig. $1 C$; Table 3). This result suggests that, regardless of IPI, JO-B neurons are equally activated during a stimulus train. Consistent with this, the peak $\left[\mathrm{Ca}^{2+}\right]_{\mathrm{i}}$ increased as IPI decreased, suggesting a simple sum- 
A

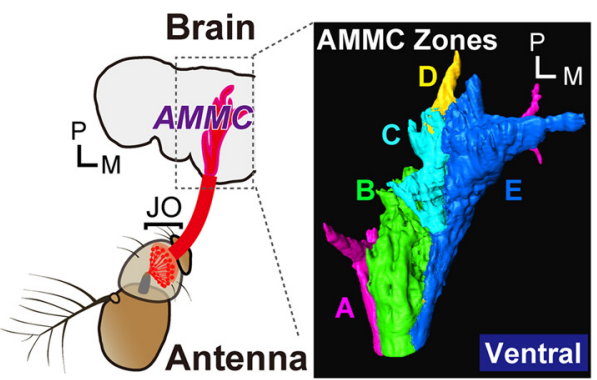

B

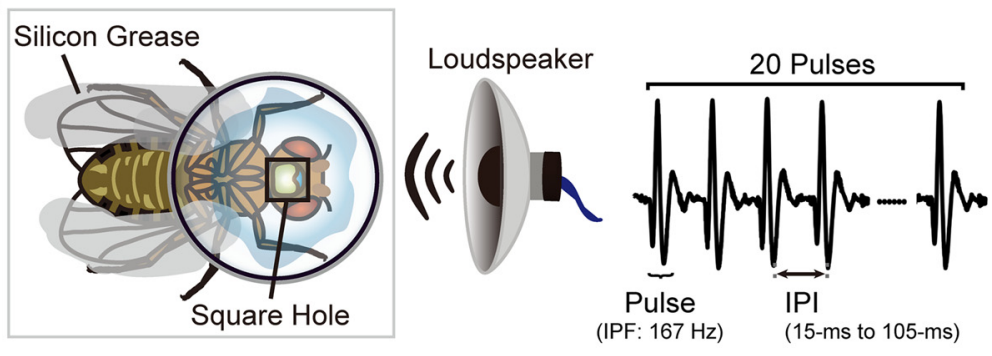

C

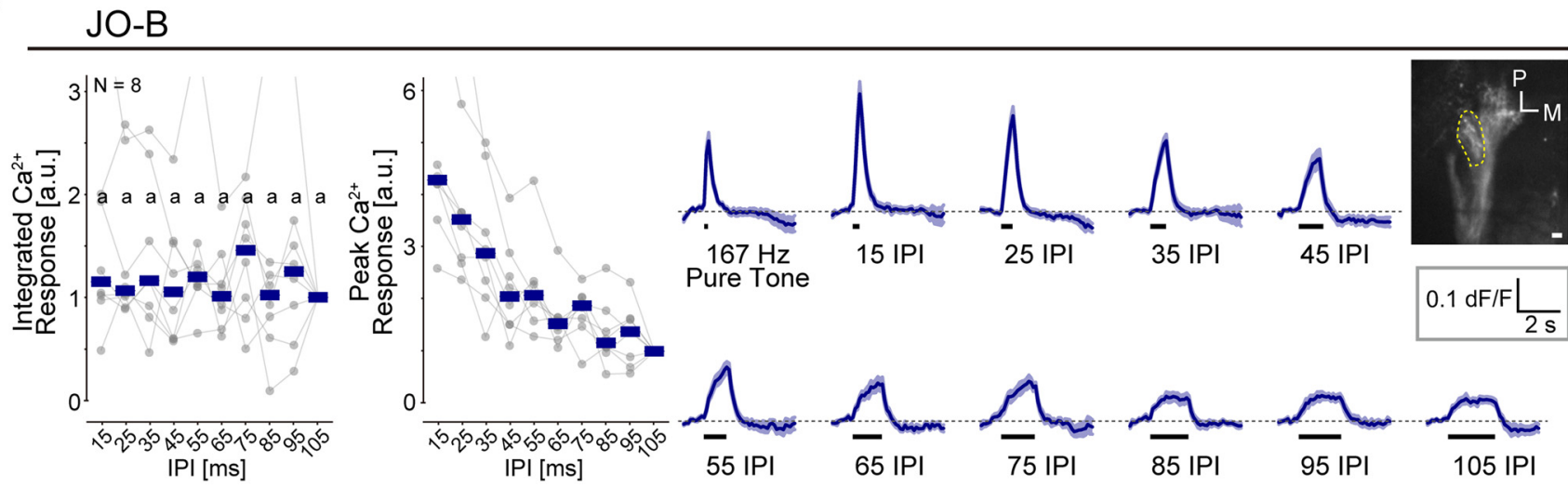

D

AMMC-B1
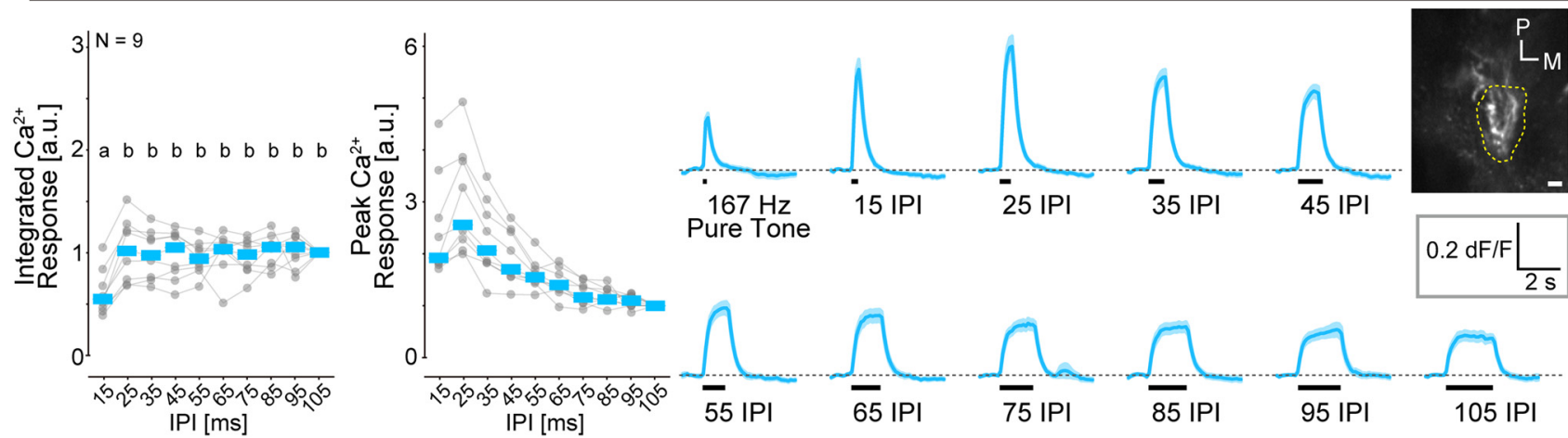

Figure 1. Different response patterns between J0-B and AMMC-B1. $A$, Neural pathway from J0 to the brain. Left, J0 neurons (red) project to the AMMC in the brain. Right, Projection targets of $J 0$ neurons in the brain. Subgroups A-E JO neurons (also called JO-A, JO-B, JO-C, JO-D, and JO-E) send their axons to a corresponding zone in the AMMC (zones A-E). JO-A and JO-B neurons are vibration sensitive, whereas JO-C and J0-E have a preference for static deflections of the antenna and JO-D responds to both vibrations and static deflections. The image was modified with permission from Matsuo et al. (2014). B, Fly preparation to monitor the $\mathrm{Ca}^{2+}$ responses in the brain. Artificial pulse songs, which comprised 20 pulse trains with different IPIs with a $167 \mathrm{~Hz}$ intrapulse frequency (IPF), were delivered from a loudspeaker. C, $\boldsymbol{D}$, Response patterns of J0-B neurons ( $\boldsymbol{C}$ ) and AMMC-B1 neurons ( $\boldsymbol{D}$ ). Left, Normalized integrated and peak $\mathrm{Ca}^{2+}$ responses for different IPIs. Each point indicates the normalized value of the $\mathrm{Ca}^{2+}$ response in each individual (see " $\mathrm{Ca}^{2+}$ imaging data analysis" section in the Materials and Methods). Data series of each individual are linked with a gray line across the IPIs. Crossbars indicate the median value of all samples to each IPI song. Right, Time traces of raw $\Delta F / F$ responses. Gray dashed lines indicate $\Delta F / F=0$. Black bars under the time trace indicate the stimulus period. Solid line and envelopes in each time trace indicate the mean and SEM, respectively, of all animals tested. Grayscale image at the right top shows an example of GCaMP6f fluorescence. The ROI for analysis of the $\mathrm{Ca}^{2+}$ response is outlined (yellow dotted line). $N$, Number of animals tested. Scale bars, $10 \mu \mathrm{m}$. M, Medial; P, posterior. Different letters indicate significant differences among groups $(p<0.05)$. a.u., Arbitrary unit. For the labeling pattern of GAL4 driver lines, see Figure 1-1 (available at https://doi.org/10.1523/JNEUROSCI.3644-17.2018.f1-1).

mation of $\mathrm{Ca}^{2+}$ transients elicited by individual stimulus pulses. These results suggest that JO-B neurons might simply transmit the information of pulse songs to downstream neurons without computing the IPI information. In contrast, the peak $\mathrm{Ca}^{2+}$ response of AMMC-B1 neurons increased monotonically as IPI decreased from 105 to $25 \mathrm{~ms}$, similar to JO-B, but exhibited a significant drop at $15 \mathrm{~ms}$ IPI (Fig. 1D; Table 3), consistent with a previous report (Vaughan et al., 2014). Integrated $\mathrm{Ca}^{2+}$ responses revealed that the activity of AMMC-B1 was similar from 105 to 25 ms IPI, but significantly smaller at $15 \mathrm{~ms}$ IPI (Fig. 1D; Table 3), suggesting that the drop of the peak $\mathrm{Ca}^{2+}$ response at $15 \mathrm{~ms}$ IPI was due to a selective attenuation of the postsynaptic activity at this IPI. This difference between JO-B and AMMC-B1 neurons suggests that the response pattern of AMMC-B1 neurons is shaped during the signal transmission from JO-B axons to the AMMC-B1 dendrites, serving as the first step in creating IPI selectivity in the song relay pathway.

Response of AMMC-B1 neurons to short IPIs are suppressed via $\mathrm{GABA}_{\mathrm{A}}$ receptors

In adult flies, GABA transmission via $G_{A B A}$ receptors suppresses the activity of olfactory projection neurons, which leads 
Table 1. Fly strains and genotypes

\begin{tabular}{|c|c|c|c|c|c|}
\hline & \multicolumn{2}{|c|}{ Driver strain } & \multicolumn{2}{|c|}{ Target neuron } & \multirow[b]{2}{*}{ Genotype } \\
\hline & GAL4 & LexA & GAL4 & LexA & \\
\hline \multicolumn{6}{|c|}{ Figure 1} \\
\hline C & F-GAL4 & & JO-B & & F-GAL4/UAS-GCaMP6f \\
\hline \multicolumn{6}{|r|}{ 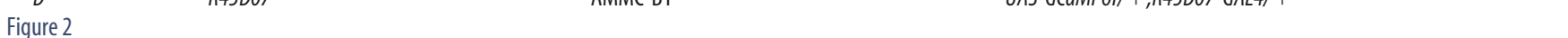 } \\
\hline \multirow{2}{*}{ A } & Rdl-GAL4 & & Rdl & & Rdl-GAL4/UAS-GFP S65T(T2) \\
\hline & & R45D07 & & AMMC-B1 & R45D07-LeXA/+,LeXAop-rCD2::GFP/+ \\
\hline \multicolumn{6}{|r|}{ 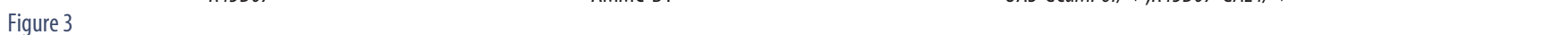 } \\
\hline \multirow[t]{2}{*}{$C$} & VT022100 & R45D07 & AMMC-LN & AMMC-B1 & R45D07-LexA/LeXAop-CD4:::spGFP11;VT022100/UAS-CD4::spGFP1-10 \\
\hline & $R 26 \mathrm{CO} 2$ & $R 45007$ & AMMC-B2 & AMMC-B1 & R45D07-LeXA/LeXAop-CD4:::SpGFP11;R26C02/UAS-CD4::SpGFP1-10 \\
\hline D & VT022100 & & AMMC-LN & & UAS-DsRed S197Y/+;UAS-nSyb::GFP/VT022100 \\
\hline \multirow[t]{2}{*}{ E } & $R 45007$ & & AMMC-B1 & & R45D07-GAL4/UAS-GCaMP6m (vk0005) \\
\hline & VT022100 & & AMMC-LN & & UAS-DsRed S197Y/+;UAS-nSyb::GFP/VT022100 \\
\hline$A$ & VT022100 & & AMMC-LN & & UAS-GCaMP6f/+;VTO22100/MKRS or TM6B \\
\hline B & $R 26 \mathrm{CO} 2$ & & AMMC-B2 & & UAS-GCaMP6f;R26CO2 \\
\hline \multicolumn{6}{|c|}{ Figure 5} \\
\hline$B$ & VT022100 & R45D07 & AMMC-LN & AMMC-B1 & R45D07-LexA/LexAop-GCaMP6f;VT022100/UAS-TNT \\
\hline & VT022100 & R45D07 & AMMC-LN & AMMC-B1 & R45D07-LexA/LexAop-GCaMP6f;VTO22100/UAS-IMPTNT \\
\hline$c$ & $R 55 \mathrm{CO} 2$ & R45D07 & AMMC-LN & AMMC-B1 & R45D07-LeXA/LeXAop-GCaMP6f;R55C02/UAS-TNT \\
\hline & $R 55 \mathrm{CO}$ & $R 45007$ & AMMC-LN & AMMC-B1 & R45D07-LexA/LeXAop-GCaMP6f;R55C02/UAS-IMPTNT \\
\hline D & $R 26 C 02$ & $R 45007$ & AMMC-B2 & AMMC-B1 & R45D07-LexA/LeXAop-GCaMP6f;R26C02/UAS-TNT \\
\hline & $R 26 C 02$ & $R 45007$ & AMMC-B2 & AMMC-B1 & R45D07-LexA/LexAop-GCaMP6f;R26C02/UAS-IMPTNT \\
\hline Figure & & & & & \\
\hline$A$ & & & & & Canton-S \\
\hline B & R45D07 & & AMMC-B1 & & UAS-TNT/+;R45D07-GAL4/+ \\
\hline & R45D07 & & AMMC-B1 & & UAS-IMPTNT/+;R45D07-GAL4/+ \\
\hline & VT017247 & & AMMC-B2 & & UAS-TNT/+;VTO17247/+ \\
\hline & VT017247 & & AMMC-B2 & & UAS-IMPTNT/+;VT017247/+ \\
\hline Figure & & & & & \\
\hline & F-GAL4 & & J0 neurons & & F-GAL4/UAS-GCaMP6f \\
\hline & R45D07 & & AMMC-B1 & & R45D07-GAL4/UAS-GCaMP6m (vk0005) \\
\hline Figure & & & & & \\
\hline & R45D07 & & AMMC-B1 & & UAS-GCaMP6f/UAS-RdI-RNAi;R45D07-GAL4/+ \\
\hline Figure & & & & & \\
\hline & & $R 45007$ & & AMMC-B1 & R45D07-LexA/LexAop-GCaMP6f \\
\hline & VT022100 & & AMMC-LN & & UAS-IVS-mCD8::RFP/+;UAS-D $\alpha 7:: G$ GFP/VT022100 \\
\hline & $R 26 C 02$ & & AMMC-B2 & & UAS-GCaMP6f;R26CO2/TM6B \\
\hline & & NV0116 & & J0 neurons & (Modified from Matsuo et al., 2016) \\
\hline Figure & & & & & \\
\hline$A$ & VT022100 & & AMMC-LN & & LexAop-CD4:.:SpGFP11/sp or CY0;UAS-CD4::SpGFP1-10/VT022100 \\
\hline & $\mathrm{R} 26 \mathrm{CO} 2$ & & AMMC-B2 & & LexAop-CD4:.:SpGFP11/Sp or CYO;UAS-CD4::SpGFP1-10/R26CO2 \\
\hline B & VT022100 & & AMMC-LN & & LexAop-CD4::SpGFP11/Sp or Cy0;UAS-CD4::SpGFP1-10/VT022100 \\
\hline & $R 26 C 02$ & & AMMC-B2 & & LexAop-CD4::SpGFP11/Sp or Cy0;UAS-CD4::SpGFP1-10/R26C02 \\
\hline Figure & & & & & \\
\hline$A$ & & $R 45 D 07$ & & AMMC-B1 & R45D07-LexA/LeXAop-GCaMP6f \\
\hline$B$ & VT022100 & R45D07 & AMMC-LN & AMMC-B1 & R45D07-LexA/LexAop-GCaMP6f;VT022100/UAS-TNT \\
\hline & VT022100 & R45D07 & AMMC-LN & AMMC-B1 & R45D07-LexA/LexAop-GCaMP6f;VT022100/UAS-IMPTNT \\
\hline & $R 55 \mathrm{CO} 2$ & R45D07 & AMMC-LN & AMMC-B1 & R45D07-LexA/LeXAop-GCaMP6f;R55C02/UAS-TNT \\
\hline & $R 55 C 02$ & R45D07 & AMMC-LN & AMMC-B1 & R45D07-LexA/LexAop-GCaMP6f;R55CO2/UAS-IMPTNT \\
\hline & $R 26 C 02$ & R45D07 & AMMC-B2 & AMMC-B1 & R45D07-LexA/LeXAop-GCaMP6f;R26C02/UAS-TNT \\
\hline & $R 26 C 02$ & R45D07 & AMMC-B2 & AMMC-B1 & R45D07-LexA/LexAop-GCaMP6f;R26C02/UAS-IMPTNT \\
\hline
\end{tabular}


Table 1. Continued

\begin{tabular}{|c|c|c|c|c|c|}
\hline & \multicolumn{2}{|c|}{ Driver strain } & \multicolumn{2}{|c|}{ Target neuron } & \multirow[b]{2}{*}{ Genotype } \\
\hline & GAL4 & LexA & GAL4 & LexA & \\
\hline \multicolumn{6}{|c|}{ Figure 6-1 } \\
\hline A & $\mathrm{R} 55 \mathrm{CO}$ & & AMMC-LN & & UAS-IVS-mCD8::GFP/+;R55CO2/+ \\
\hline \multirow[t]{2}{*}{$B$} & VT021793 & & AMMC-B2 & & (Modified from Matsuo et al., 2016) \\
\hline & VT017247 & & AMMC-B2 & & (Modified from Matsuo et al., 2016) \\
\hline \multicolumn{6}{|c|}{ Figure 6-2 } \\
\hline & & & & & Canton-S \\
\hline & & & & & UAS-IMPTNT/+;R45D07-GAL4/+ \\
\hline & & & & & UAS-IMPTNT/+;VT022100/+ \\
\hline & & & & & UAS-IMPTNT/+;R55CO2/+ \\
\hline & & & & & UAS-IMPTNT/+;R26CO2/+ \\
\hline & & & & & UAS-IMPTNT/+,VT021793/+ \\
\hline & & & & & UAS-IMPTNT/+;VT017247/+ \\
\hline
\end{tabular}

to shape their odor responses (Wilson and Laurent, 2005). Because the response of AMMC-B1 neurons appeared to be attenuated at 15 ms IPI (Fig. $1 D$ ), we speculated that GABAergic inputs via $\mathrm{GABA}_{\mathrm{A}}$ receptors in AMMC-B1 neurons might suppress the IPI responses of AMMC-B1 neurons. We analyzed whether Rdl, a subunit of the $\mathrm{GABA}_{\mathrm{A}}$ receptor (Hosie et al., 1997), was expressed in AMMC-B1 neurons by using $R d l$-GAL4 strain (Kolodziejczyk et al., 2008; Yuan et al., 2014), in which GAL4 expression is driven by the upstream regulatory region of $R d l$ (Fig. $2 A$ ). We found $R d l$-GAL4 labeled AMMC-B1 neurons in both female and male brains $(n=$ 3 ), suggesting that AMMC-B1 neurons express Rdl. We then knocked down the expression of Rdl in AMMC-B1 neurons of female flies and evaluated their response pattern. Rdl knockdown flies had a significantly increased raw $\Delta F / F$ response amplitude to IPIs between 15 and $35 \mathrm{~ms}$ in both the integrated and peak responses, but not to IPIs longer than $55 \mathrm{~ms}$ (Fig. 2B; Fig. 2-1, available at https://doi.org/10.1523/JNEUROSCI.3644-17. 2018.f2-1; Table 2). These results indicate that the AMMC-B1 activity was suppressed particularly between 15 and 35 ms IPIs by GABAergic inputs via $\mathrm{GABA}_{\mathrm{A}}$ receptors.

To compare the response patterns of knock-down and control flies with these short IPIs, we normalized the integrated responses by that of the reference response (i.e., $105 \mathrm{~ms}$ IPI). The response pattern of knock-down flies was statistically different from that of control flies (Fig. 2C; Table 2). Rdl knock-down flies responded to $15 \mathrm{~ms}$ IPI as strongly as to $105 \mathrm{~ms}$ IPI, whereas control flies responded much weaker to the $15 \mathrm{~ms}$ IPI than to the $105 \mathrm{~ms}$ IPI (Fig. 2C; Table 3). In addition to this change, responses to 25 and $35 \mathrm{~ms}$ IPIs were also increased in knock-down flies (Fig. 2C; Table 3). Rdl knock-down flies responded to 25 and 35 ms IPIs more than to the $105 \mathrm{~ms}$ IPI, whereas control flies responded to these IPIs at the same level. Altogether, these results suggest that AMMC-B1 receives GABAergic inputs via $\mathrm{GABA}_{\mathrm{A}}$ receptors, which suppress the response of AMMC-B1 neurons at short IPIs.

\section{GABAergic local interneurons form a circuit with JO-B and AMMC-B1 neurons}

The Rdl knock-down results suggest the possibility that unknown inhibitory GABAergic neurons suppress the response of AMMC-B1 neurons to pulse songs with short IPIs. To identify the responsible neurons, we focused on two GABAergic local interneurons, AMMC-LN and AMMC-B2, which innervate the AMMC zone B, where JO-B axons and AMMC-B1 dendritic regions are located (Kamikouchi et al., 2006; Lai et al., 2012; Tootoonian et al., 2012; Vaughan et al., 2014; Matsuo et al., 2016) (Fig. 3A). AMMC-LN neurons innervate the ipsilateral AMMC zones B and D and are involved in courtship hearing in both sexes (Vaughan et al., 2014;
Matsuo et al., 2016). AMMC-B2 neurons have input sites in the ipsilateral $\mathrm{AMMC}$ zones $\mathrm{B}$ and $\mathrm{E}$ and output sites in the contralateral AMMC zone B (Matsuo et al., 2016). AMMC-B2 neurons are activated by sound, but their functional role remains largely unknown (Tootoonian et al., 2012). Therefore, we hypothesized that these GABAergic local interneurons contribute to shape the response of AMMC-B1 neurons.

We first investigated whether AMMC-LN and AMMC-B2 neurons have synaptic connections with AMMC-B1 neurons. Coregistration of single AMMC-B1, AMMC-B2, and AMMC-LN neurons on a template brain revealed their spatial overlap (Fig. $3 B$ ), implying a synaptic connection between each local interneuron and the AMMC-B1 neurons. To estimate the synaptic contact, we used the GRASP technique (Feinberg et al., 2008) (for fly genotypes, see Table 1) and found GRASP signals between AMMC-LN/ AMMC-B1 and AMMC-B2/AMMC-B1 in the AMMC (Fig. 3C; $n=$ 4 for AMMC-LN/AMMC-B1 and $n=8$ for AMMC-B2/AMMC$\mathrm{B} 1)$. We confirmed that anti-CD4 antibodies, which recognize both CD4::spGFP1-10 and CD4::spGFP11 fragments, detected AMMC-B1, AMMC-LN, and AMMC-B2 neurons, whereas the CD4::spGFP1-10 fragment recognized by chicken anti-GFP antibodies was detected only in AMMC-LN and AMMC-B2 neurons (Fig. 3C; see Materials and Methods for antibodies used). These results are consistent with the scenario that each of these GABAergic local interneurons has synaptic contacts with AMMC-B1 neurons.

We then examined the direction of the information flow between AMMC-B1 neurons and each GABAergic local interneuron. When we expressed a presynaptic marker, nSyb::GFP, in AMMC-LN neurons, the nSyb::GFP signals were distributed in the AMMC zone B, but not in the AMMC zone D (Fig. 3D; $n=$ 3). This indicates that AMMC-LN has output sites in AMMC zone B. Likewise, the presynaptic sites of the AMMC-B2 were localized in AMMC zone B (Matsuo et al., 2016). Coregistration on a template brain demonstrated that the presynaptic sites of both AMMC-LN and AMMC-B2 spatially overlapped with the dendritic region of AMMC-B1 (Fig. 3E) (Lai et al., 2012; Vaughan et al., 2014). These results strongly suggest that AMMC-LN and AMMC-B2 send signals directly to AMMC-B1 neurons. Note that the presynaptic sites of AMMC-LN (Fig. 3D) and AMMC-B2 (Matsuo et al., 2016) are seemingly distributed more broadly than their GRASP signals with AMMC-B1 (Fig. 3C), suggesting that other downstream neurons also receive input from these GABAergic local interneurons.

Because AMMC-LN and AMMC-B2 innervate AMMC, where JO neurons project (Matsuo et al., 2016), we next investigated whether AMMC-LN and AMMC-B2 receive inputs from 
Table 2. Statistical comparison of the response pattern to a series of IPIs between different neuronal types/conditions

\begin{tabular}{|c|c|c|c|c|c|c|c|c|c|c|}
\hline & $\begin{array}{l}\text { Neuronal types/ } \\
\text { conditions }\end{array}$ & Data & Statistical method & & Df & Sum Sq & Mean Sq & $F$-value & $p$-value & Significance \\
\hline \multirow[t]{5}{*}{ Figure $1 C, D$} & J0-B vs AMMC-B1 & Integrated $\mathrm{Ca}^{2+}$ response & Two-way ANOVA with repeated measures & Strain & 1 & 145 & 145 & 34.7 & $2.98 \mathrm{E}-05$ & $* * *$ \\
\hline & & & & Residuals & 15 & 62.9 & 4.19 & & & \\
\hline & & & & IPI & 9 & 6.56 & 0.73 & 2.68 & $6.85 \mathrm{E}-03$ & $* *$ \\
\hline & & & & Interaction & 9 & 12.0 & 1.33 & 4.89 & $1.12 \mathrm{E}-05$ & $* * *$ \\
\hline & & & & Residuals & 135 & 36.8 & 0.273 & & & \\
\hline \multirow[t]{5}{*}{ Figure $2 C$} & Rdl-RNAi vs Control & Integrated $\mathrm{Ca}^{2+}$ response & Two-way ANOVA with repeated measures & Strain & 1 & 53.4 & 53.4 & 8.6 & $8.80 \mathrm{E}-03$ & ** \\
\hline & & & & Residuals & 18 & 111 & 6.19 & & & \\
\hline & & & & IPI & 9 & 25.3 & 2.81 & 14.7 & $<2 \mathrm{E}-16$ & $* * *$ \\
\hline & & & & Interaction & 9 & 10.91 & 1.21 & 6.34 & $1.11 \mathrm{E}-07$ & $* * *$ \\
\hline & & & & Residuals & 162 & 31.0 & 0.191 & & & \\
\hline \multirow[t]{5}{*}{ Figure $5 B$} & TNT vs IMPTNT & Integrated $\mathrm{Ca}^{2+}$ response & Two-way ANOVA with repeated measures & Strain & 1 & 1.03 & 1.03 & 0.318 & 0.578 & \\
\hline & & & & Residuals & 24 & 77.7 & 3.24 & & & \\
\hline & & & & $\mid \mathrm{PI}$ & 9 & 1.69 & 0.187 & 4.77 & $8.43 \mathrm{E}-06$ & $* * *$ \\
\hline & & & & Interaction & 9 & 0.920 & 0.102 & 2.60 & 7.19E-03 & ** \\
\hline & & & & Residuals & 216 & 8.48 & 0.0393 & & & \\
\hline \multirow[t]{5}{*}{ Figure $5 C$} & TNT vs IMPTNT & Integrated $\mathrm{Ca}^{2+}$ response & Two-way ANOVA with repeated measures & Strain & 1 & 0.0100 & $9.40 \mathrm{E}-03$ & $4.00 \mathrm{E}-03$ & 0.949 & \\
\hline & & & & Residuals & 25 & 56.4 & 2.26 & & & \\
\hline & & & & IPI & 9 & 3.34 & 0.371 & 4.35 & $3.08 \mathrm{E}-05$ & $* * *$ \\
\hline & & & & Interaction & 9 & 1.61 & 0.179 & 2.10 & 0.0308 & $*$ \\
\hline & & & & Residuals & 225 & 19.2 & 0.0854 & & & \\
\hline \multirow[t]{6}{*}{ Figure 5D } & TNT vs IMPTNT & Integrated $\mathrm{Ca}^{2+}$ response & Two-way ANOVA with repeated measures & Strain & 1 & 0.510 & 0.506 & 0.210 & 0.651 & \\
\hline & & & & Residuals & 24 & 57.9 & 2.41 & & & \\
\hline & & & & IPI & 9 & 1.62 & 0.180 & 3.83 & $1.65 \mathrm{E}-04$ & $* * *$ \\
\hline & & & & Interaction & 9 & 0.503 & 0.0559 & 1.19 & 0.305 & \\
\hline & & & & Residuals & 216 & 10.2 & 0.0471 & & & \\
\hline & & Data & Statistical method & IPI & Sample size & U & $p$-value & Adjusted $p$ - & -value & \\
\hline \multirow[t]{20}{*}{ Figure $2 B$} & Rdl-RNAi vs Control & $\begin{array}{l}\text { Integrated } \mathrm{Ca}^{2+} \text { response } \\
\qquad(\text { Raw df/F) }\end{array}$ & $\begin{array}{l}\text { Mann-Whitney } U \text { test followed by } \\
\text { Bonferroni multiple comparisons }\end{array}$ & 15 & 9,11 & 4 & $5.47 \mathrm{E}-04$ & 5.47E-03 & & $* *$ \\
\hline & & & & 25 & 9,11 & 8 & $1.62 \mathrm{E}-03$ & 0.0162 & & $*$ \\
\hline & & & & 35 & 9,11 & 9 & $2.09 \mathrm{E}-03$ & 0.0209 & & $*$ \\
\hline & & & & 45 & 9,11 & 17 & 0.0135 & 0.135 & & \\
\hline & & & & 55 & 9,11 & 17 & 0.0135 & 0.135 & & \\
\hline & & & & 65 & 9,11 & 31 & 0.160 & 1.00 & & \\
\hline & & & & 75 & 9,11 & 22 & 0.0367 & 0.367 & & \\
\hline & & & & 85 & 9,11 & 34 & 0.239 & 1.00 & & \\
\hline & & & & 95 & 9,11 & 33 & 0.210 & 1.00 & & \\
\hline & & & & 105 & 9,11 & 32 & 0.184 & 1.00 & & \\
\hline & & $\begin{array}{l}\text { Peak } \mathrm{Ca}^{2+} \text { response } \\
\quad(\text { Raw df/F) }\end{array}$ & $\begin{array}{l}\text { Mann-Whitney U test followed by } \\
\text { Bonferroni multiple comparisons }\end{array}$ & 15 & 9,11 & 2 & $3.08 \mathrm{E}-04$ & $3.08 \mathrm{E}-03$ & & $* *$ \\
\hline & & & & 25 & 9,11 & 7 & $1.24 \mathrm{E}-03$ & 0.0124 & & * \\
\hline & & & & 35 & 9,11 & 7 & $1.24 \mathrm{E}-03$ & 0.0124 & & $*$ \\
\hline & & & & 45 & 9,11 & 11 & $3.44 \mathrm{E}-03$ & 0.0344 & & $*$ \\
\hline & & & & 55 & 9,11 & 15 & $8.76 \mathrm{E}-03$ & 0.0876 & & \\
\hline & & & & 65 & 9,11 & 24 & 0.0527 & 0.527 & & \\
\hline & & & & 75 & 9,11 & 16 & 0.0109 & 0.109 & & \\
\hline & & & & 85 & 9,11 & 35 & 0.271 & 1.00 & & \\
\hline & & & & 95 & 9,11 & 33 & 0.210 & 1.00 & & \\
\hline & & & & 105 & 9,11 & 33 & 0.210 & 1.00 & & \\
\hline
\end{tabular}

Asterisks indicate statistical significance: ${ }^{* *} p<0.001,{ }^{* *} p<0.01,{ }^{*} p<0.05$.

Df, Degree of freedom; Sq, square.

JO neurons. Because most JO neurons use acetylcholine as a neurotransmitter (Ishikawa et al., 2017), we expressed a postsynaptic marker encoding GFP-tagged nicotinic acetylcholine receptor $\mathrm{D} \alpha 7$ (D $\alpha 7:: \mathrm{GFP})$ (Leiss et al., 2009) in AMMC-LN neurons. Signals of $\mathrm{D} \alpha 7:: \mathrm{GFP}$ expressed in AMMC-LN were detected in AMMC zones B and D (Fig. $3 F ; n=5$ ), which are innervated by axons of subgroup B and D JO neurons (JO-B and JO-D), implying that AMMC-LN had synaptic contacts with JO neurons. We reported previously that AMMC-B2 also has postsynaptic sites in AMMC zones B and E (Matsuo et al., 2016). Indeed, GRASP signals of both JO neurons/AMMC-LN and JO neurons/ AMMC-B2 were observed in AMMC (Fig. $3 G ; n=3$ for JO
neurons/AMMC-LN and $n=5$ for JO neurons/AMMC-B2). Conversely, control flies, in which only CD4::spGFP1-10 was expressed, showed no GRASP signals even though the CD4:: spGFP1-10 signals were detected (Fig. 3-2, available at https:// doi.org/10.1523/JNEUROSCI.3644-17.2018.f3-2). These results strongly suggest that JO-B neurons transmit signals directly to these two GABAergic local interneurons through cholinergic synapses.

Together, our data suggest that GABAergic AMMC-LN and AMMC-B2 neurons form feedforward pathways, presumably inhibitory, which transmit signals from JO-B neurons to AMMC-B1 neurons. 
Table 3. Statistical comparison of the $\mathrm{Ca}^{2+}$ responses to a series of IPIs in each GAL4 strain or condition

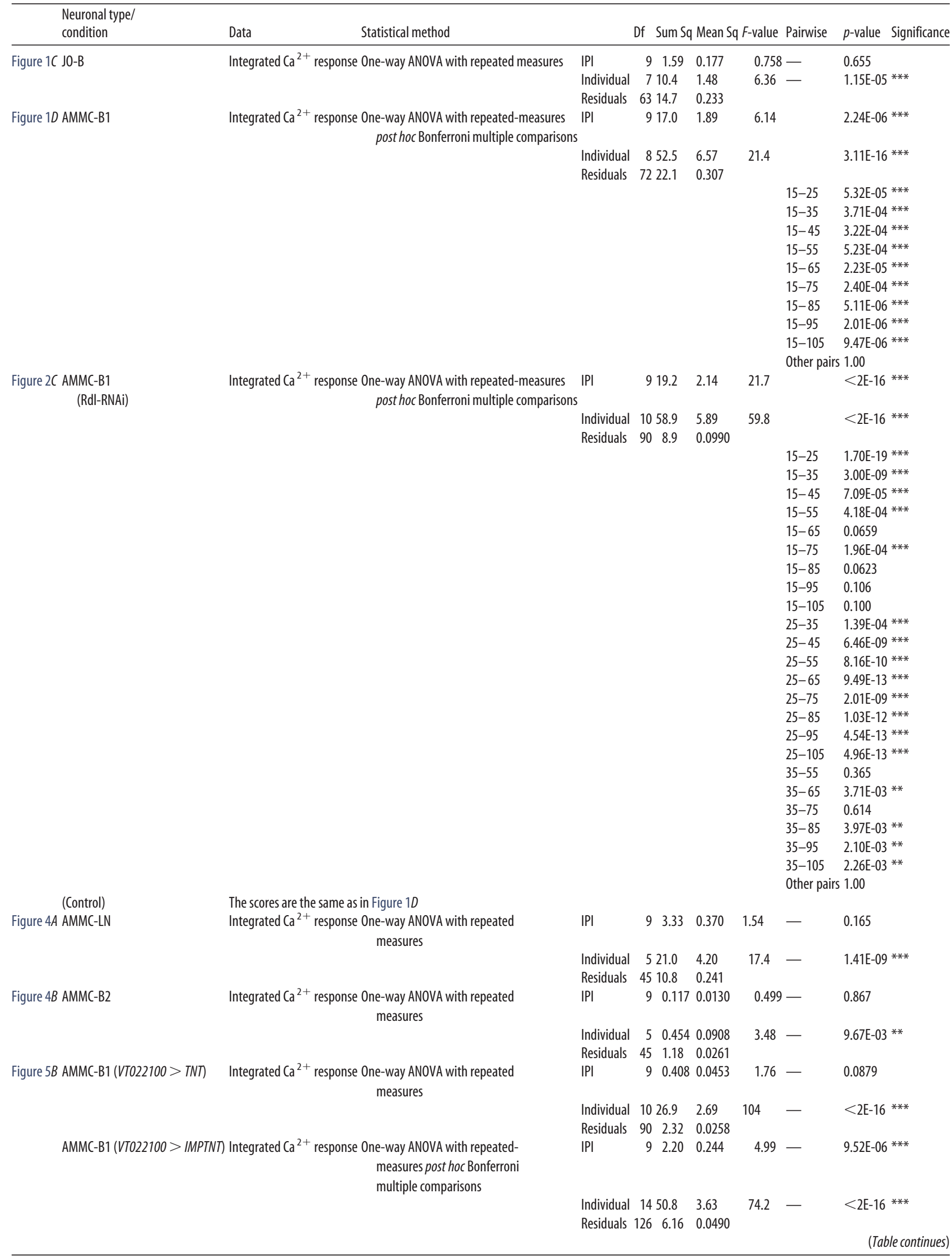


Table 3. Continued

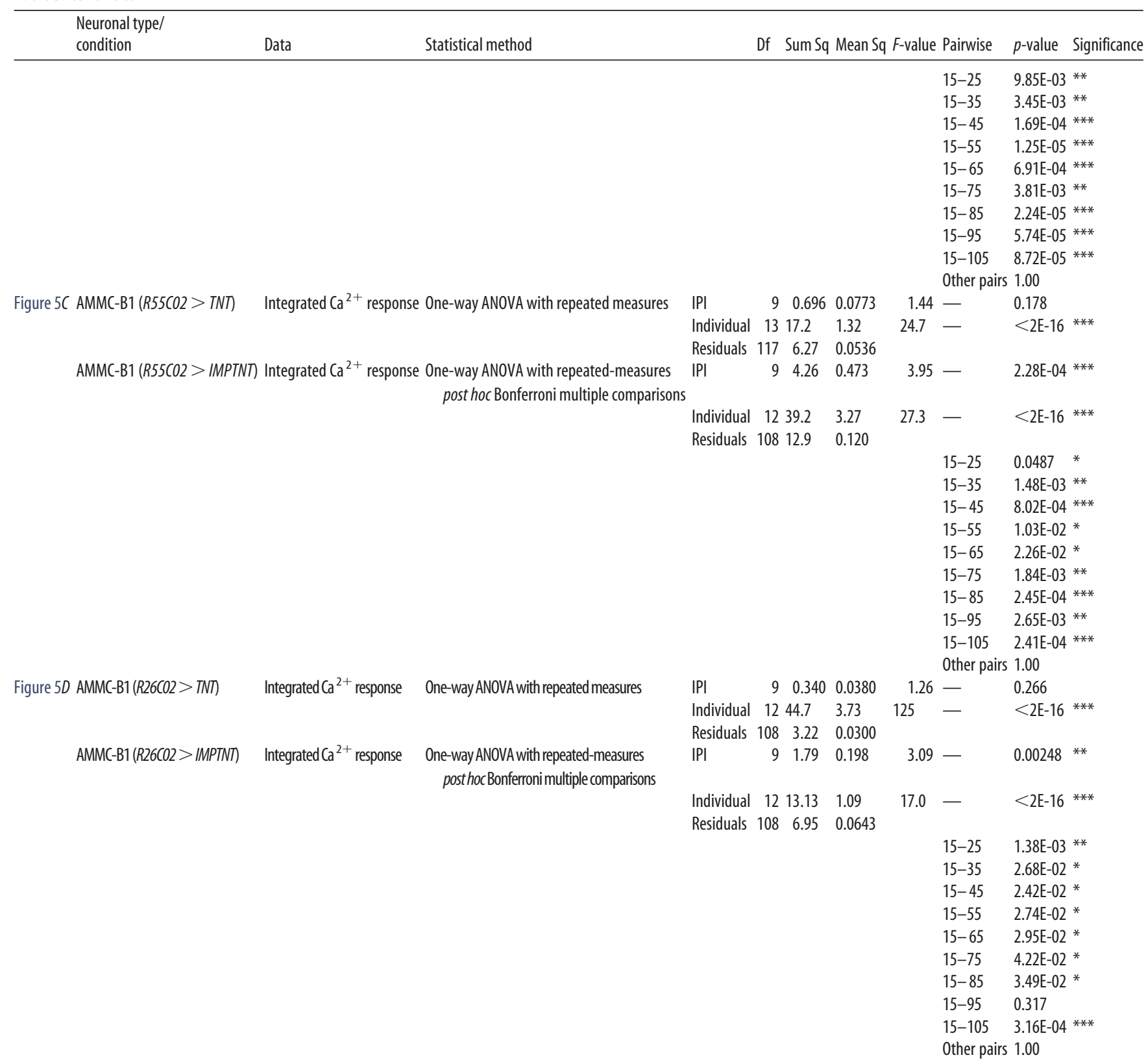

Asterisks indicate statistical significance: ${ }^{* * *} p<0.001,{ }^{* *} p<0.01,{ }^{*} p<0.05$. For a statistical comparison of the $\mathrm{Ca}^{2+}$ responses to a series of IPIs in each condition of AMMC-B1 neurons labeled by the 845007 -LexA driver, see Table 3-1 (available at https://doi.org/10.1523/JNEUROSCI.3644-17.2018.t3-1).

Df, Degree of freedom; Sq, square.

\section{GABAergic local interneurons shape the AMMC-B1 response pattern}

To characterize the response pattern of AMMC-LN and AMMC-B2 neurons, we measured their $\mathrm{Ca}^{2+}$ responses to a series of IPIs in female flies. We monitored their activity in the AMMC, in which both interneurons have presynaptic and postsynaptic sites (Fig. 3; Matsuo et al., 2016). Both neurons showed $\mathrm{Ca}^{2+}$ responses to all the pulse songs tested (Fig. 4). The response pattern of these neurons was flat to all IPIs between 15 and $105 \mathrm{~ms}$ in the integrated $\mathrm{Ca}^{2+}$ response (Fig. 4; Table 3). The peak $\mathrm{Ca}^{2+}$ response increased monotonically as the IPI decreased from 105 to $15 \mathrm{~ms}$ (Fig. 4), resembling the JO-B neurons and contrasting with the AMMC-B1 neurons (Fig. 1C,D).

To test directly whether the AMMC-LN and AMMC-B2 activities shape the response pattern of AMMC-B1 neurons to IPI length, we examined the AMMC-B1 responses to varying IPIs after silencing the synaptic transmission of either AMMC-LN or AMMC-B2. To this end, we combined GAL4/UAS and LexA/ lexAop systems to express TNT (Sweeney et al., 1995) in either AMMC-LN or AMMC-B2 and GCaMP6f in AMMC-B1 neurons, respectively (Fig. 5A; see Table 1 for fly genotypes). To compare the response patterns of silenced and control flies across IPIs, we normalized the $\mathrm{Ca}^{2+}$ responses to the reference IPI response (i.e., $105 \mathrm{~ms}$ IPI; Fig. 5B-D, left). When we silenced either AMMC-LN or AMMC-B2, the integrated $\mathrm{Ca}^{2+}$ response of AMMC-B1 was similar among all IPIs tested and the peak $\mathrm{Ca}^{2+}$ response increased monotonically as IPI decreased (Fig. $5 B-D$, left, red; Table 3). Conversely, control flies expressing an inactive form of TNT (IMPTNT) decreased the response at $15 \mathrm{~ms}$ IPI (Fig. 5B-D, left, blue; Table 3), as was observed in intact AMMC-B1 (Fig. 1D). These results indicate 
A

Female
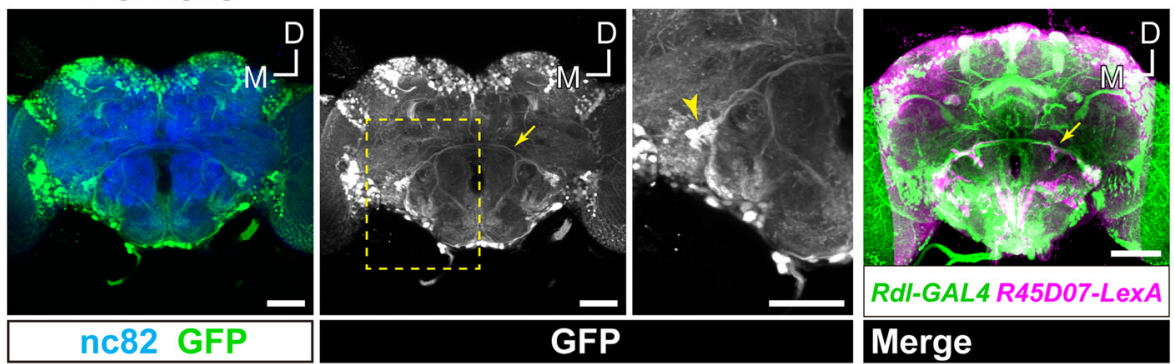

Male
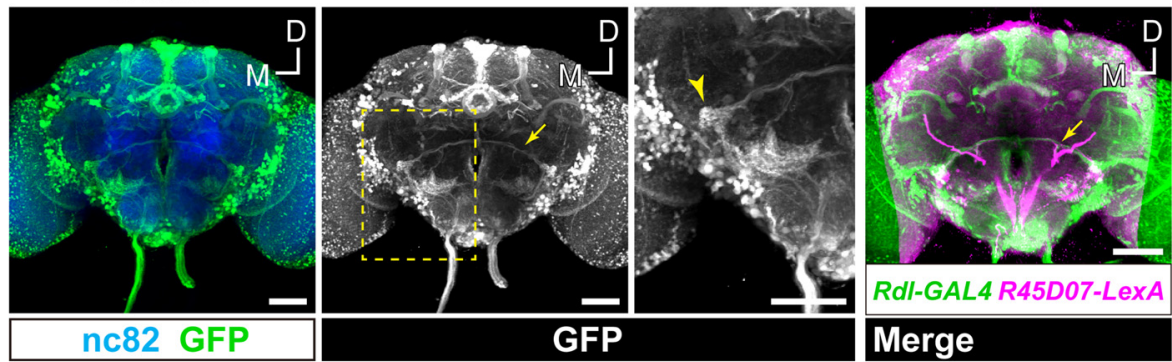

B

\section{AMMC-B1 (Rdl Knockdown)}
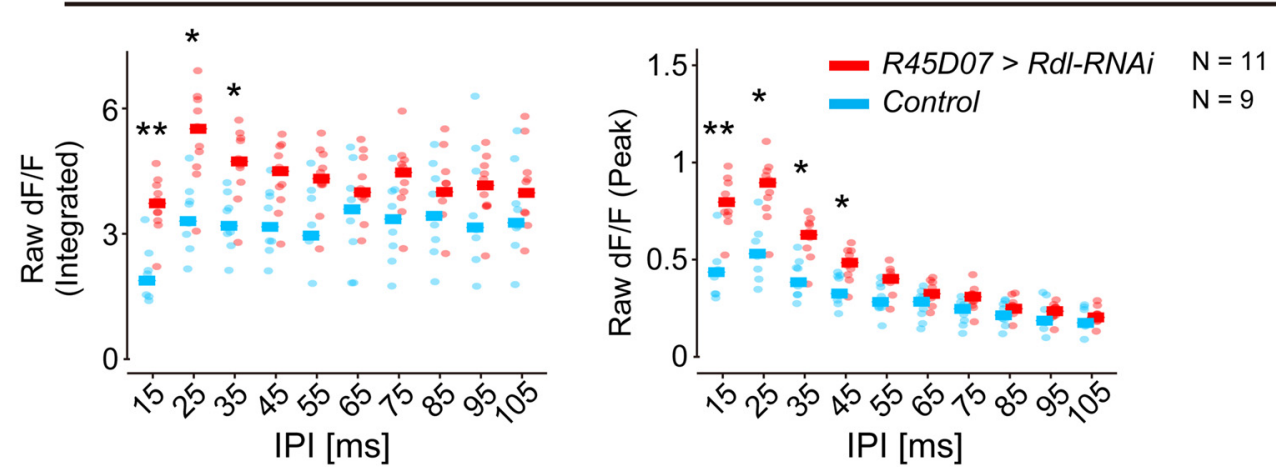

C
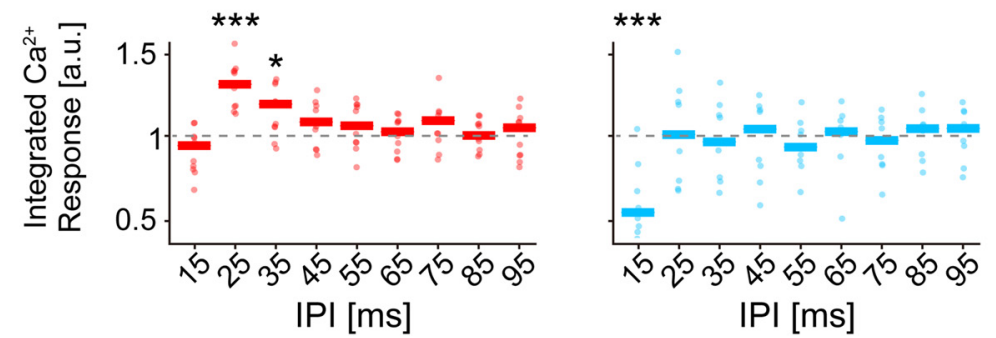

Figure 2. AMMC-B1 neurons receive GABAergic inputs via GABA $A_{A}$ receptors. $A$, Expression pattern of the $R d l-G A L 4$ driver in female and male brains. GFP expressed by $R d l-G A L 4$ driver (green) are shown. Left, Blue signals show neuropil visualized using the nc 82 antibody. Middle, White signals show the labeled neurons visualized using anti-GFP antibodies. The brain is the same as that shown on the left. Arrows indicate AMMC-B1 neurons, which project from the AMMC zone B to the wedge (WED). Yellow dotted frame indicates the position for a magnified view. Arrowheads in the magnified view indicate the AMMC-B1 neurons innervating the WED. Right, Coregistration of Rdl-GAL4 and R45D07-LexA expressions. Overlay of registered R45D07-LexA (magenta) and Rdl-GAL4 (green) brains visualized an overlap in AMMC-B1 neurons (arrows). Stacked optical sections are shown. Scale bars, $50 \mu \mathrm{m}$. D, Dorsal; M, medial. B, Raw $\triangle F / F$ responses of AMMC-B1 neurons in Rdl knock-down (red) and control (light blue) flies. C, Normalized Ca ${ }^{2+}$ responses of AMMC-B1 neurons in Rdl knock-down (red) and control (light blue) flies. $\mathrm{Ca}^{2+}$ response is normalized by the response to a $105 \mathrm{~ms} \mathrm{IPI} \mathrm{(reference} \mathrm{stimulus)} \mathrm{to} \mathrm{compare} \mathrm{the} \mathrm{response} \mathrm{patterns.} \mathrm{Ca}^{2+}$ responses are displayed as in Figure 1. Gray dashed lines in Cindicate the response to the reference stimulus. Number of animals tested is denoted in $\boldsymbol{B}$. The data of AMMC-B1 (control) are the same as those shown in Figure 1D. Asterisks indicate statistical differences: ${ }^{*} p<0.05$; ${ }^{* *} p<0.01 ;{ }^{* * *} p<0.001$. a.u., Arbitrary unit. For the time traces of raw $\Delta$ F/F responses of AMMC-B1 neurons in Rdl knock-down flies, see Figure 2-1 (available at https://doi.org/10.1523/JNEUROSCI.3644-17.2018.f2-1).

that AMMC-LN and AMMC-B2 shape the response pattern of AMMC-B1 neurons by suppressing them at $15 \mathrm{~ms}$ IPI.

It should be noted that, although the raw $\Delta F / F$ response of AMMC-B1 neurons at $15 \mathrm{~ms}$ IPI tended to increase when each
GABAergic interneuron was silenced, the overall responses to varying IPIs was not increased significantly in the silenced flies (Fig. 5-1 $B$, available at https://doi.org/10.1523/JNEUROSCI. 3644-17.2018.f5-1; Fig. 5B-D, right; see TNT vs IMPTNT in 
A
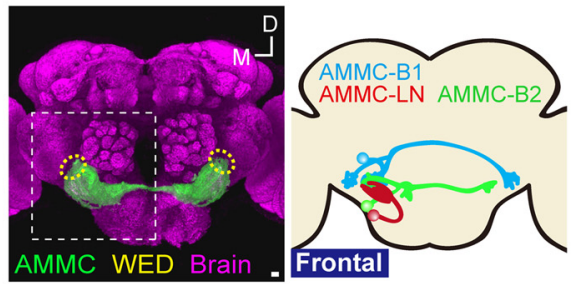

C
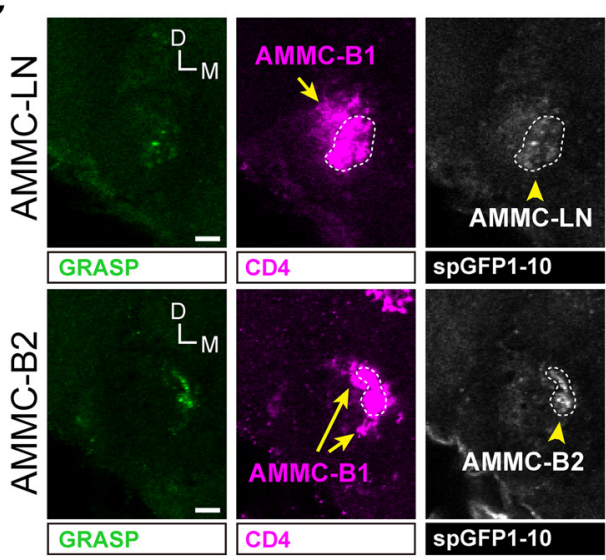

E
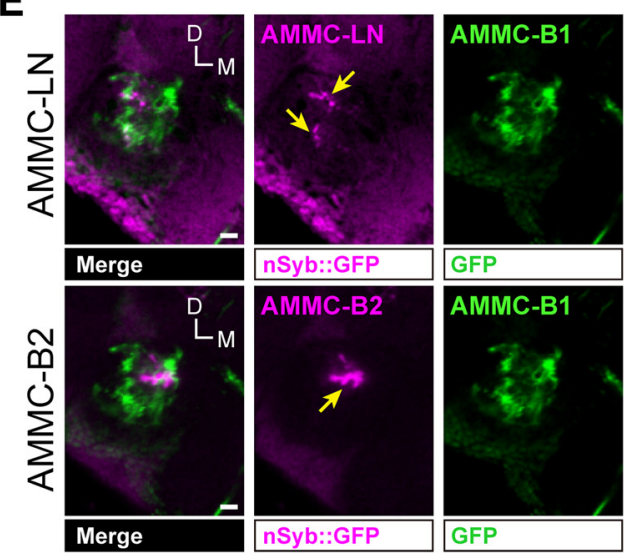

\section{G}
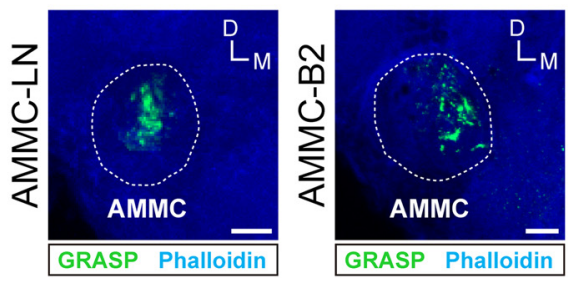

B

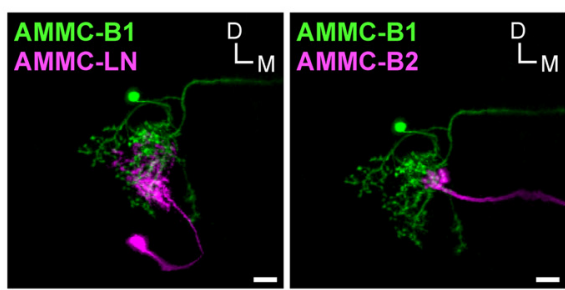

D

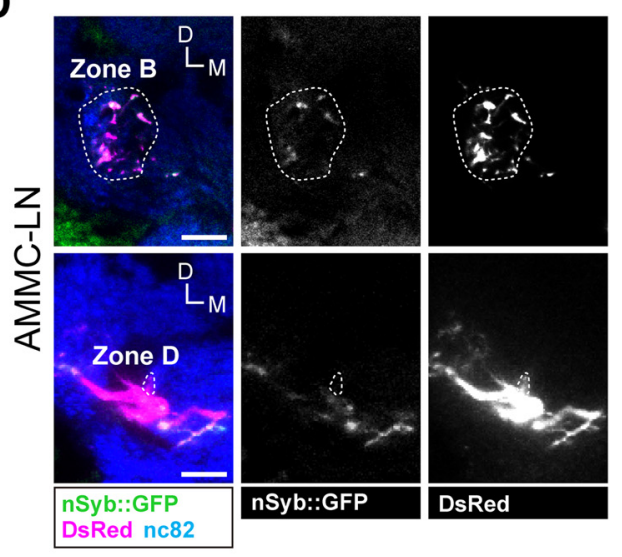

F

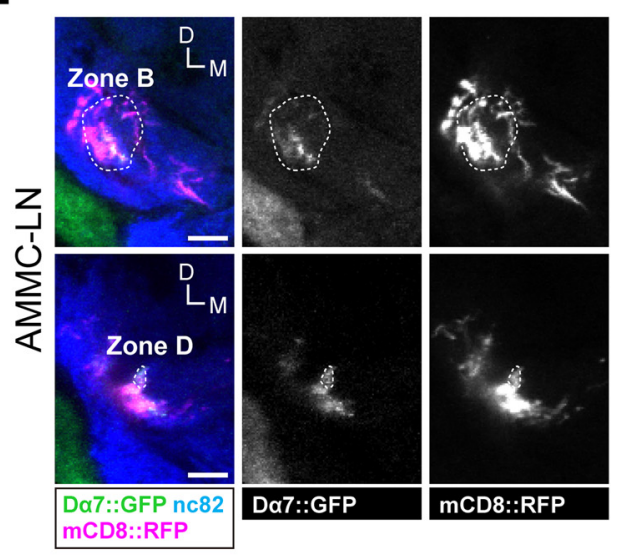

Figure 3. Neural circuits comprised of two GABAergic local interneurons. A, Auditory neurons in the brain. Left, Auditory centers in the fly brain. AMMC (green) and wedge (WED, outlined in yellow dotted line) represent the primary auditory center and a major secondary auditory center, respectively. White dotted frame indicates the position for $\boldsymbol{B}-\boldsymbol{G}$. The image was modified with permission from Matsuo et al. (2014). Right, Schematic representation of single AMMC-B1 (light blue), AMMC-LN (red), and AMMC-B2 (green) neurons in a fly brain. AMMC-B1 neurons project from the ipsilateral AMMC zone B to the WED of both hemispheres. B, Spatial distribution of AMMC-B1 and each local interneuron. AMMC-B1 (green) and each local interneuron (magenta) were registered on a template brain. The images of the single neurons were modified with permission from Matsuo et al. (2016). C, Synaptic connections between AMMC-B1 neurons and each local interneuron. GRASP signals (green), both CD4::spGFP fragments expressed in AMMC-B1 and each local interneuron (magenta), and each local interneuron expressing CD4::spGFP1-10 (white) are shown. White dots outline the region where CD4::spGFP1-10 signals of each local interneuron are distributed. Arrows indicate signals of AMMC-B1 neurons. D, Output sites of AMMC-LN neurons. Dashed line indicates the AMMC zones B and D. Output sites of AMMC-LN neurons were labeled by nSyb::GFP presynaptic maker (green). AMMC-LN neurons were labeled by DsRed (magenta). Blue signals show neuropil visualized using the $n c 82$ antibody. Top and bottom panels show single and stacked optical sections, respectively, in different depths of the brain. $E$, Spatial distribution of the presynaptic sites of two local interneurons and the dendritic region of AMMC-B1 neurons. Dendritic region of AMMC-B1 neurons (green) and presynaptic sites of each local interneuron (magenta) were registered on a template brain. Arrows indicate the presynaptic marker nSyb::GFP expressed in AMMC-LN and AMMC-B2.F, Input sites of AMMC-LN neurons. Dashed line outlines the AMMC zones B and D. Input sites labeled by the postsynaptic marker D $\alpha 7:$ GFP (green) and AMMC-LN neurons labeled by mCD8::RFP (magenta) are shown. Blue signals show neuropil visualized using the nc82 antibody. Stacked optical sections are shown in different depth of the brain. G, Synaptic connections between J0 neurons and each local interneuron. Green signals show GRASP signals. Phalloidin labeling visualizes the contour of the brain (blue). Scale bars, $10 \mu \mathrm{m}(\boldsymbol{A}-\mathbf{G})$. D, Dorsal; M, medial. For the labeling pattern of GAL4 and LexA driver lines, see Figure 3-1 (available at https://doi.org/10.1523/JNEUROSCI.3644-17. 2018.f3-1). For the validation of GRASP signals between J0 neurons and each local interneuron, see Figure 3-2 (available at https://doi.org/10.1523/JNEUROSCI.3644-17.2018.f3-2). 
A AMMC-LN
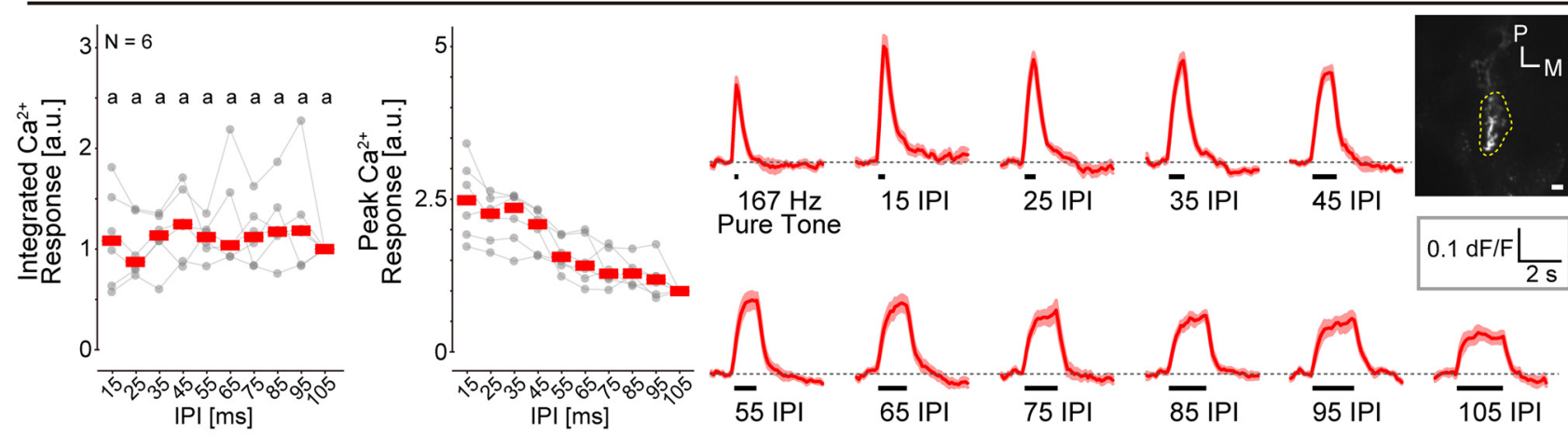

$0.1 \mathrm{dF} / \mathrm{F} \frac{}{2 \mathrm{~s}}$
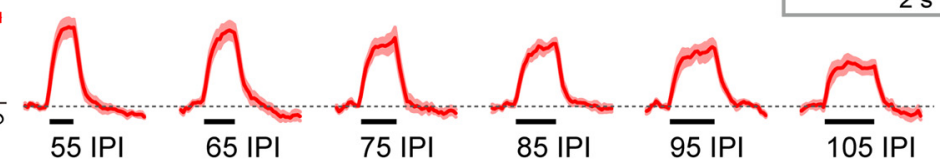

B

AMMC-B2
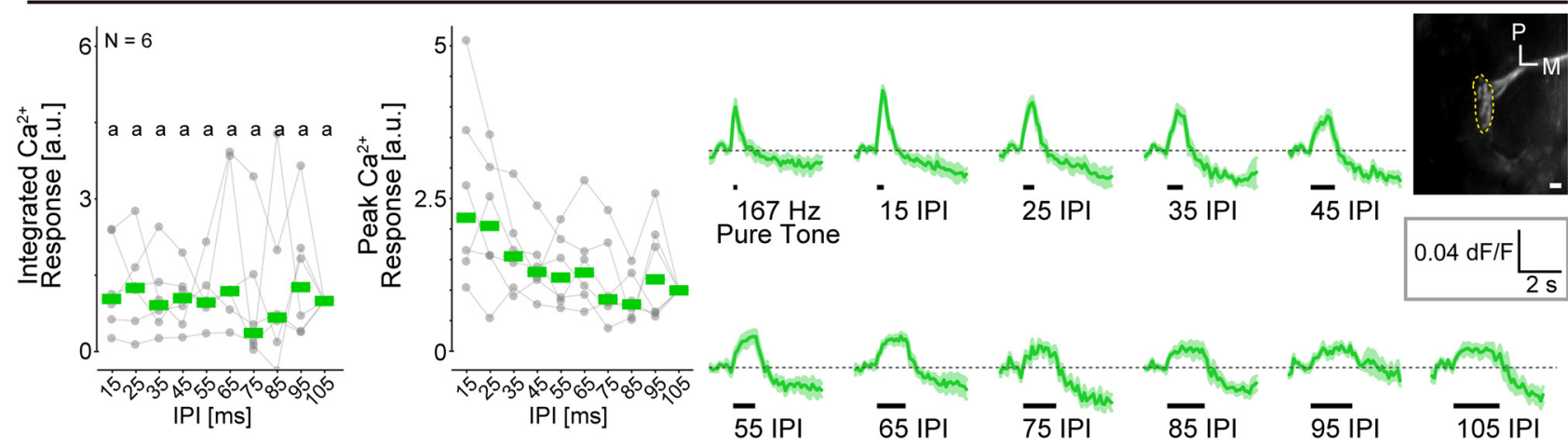

Figure 4. Response pattern of each GABAergic local interneuron. AMMC-LN $(\boldsymbol{A})$ and AMMC-B2 (B) neurons are shown. The $\mathrm{Ca}^{2+}$ response is normalized to the response to $105 \mathrm{~ms} I \mathrm{IPI}($ reference stimulus) to compare the response patterns across flies (left). Normalized $\mathrm{Ca}^{2+}$ responses are displayed as in Figure 1. Raw $\Delta F / F$ responses are shown in the time trace (right). $N$, Number of animals tested. Scale bars, $10 \mu \mathrm{m}$. M, Medial; P, posterior.

Table 3-1, available at https://doi.org/10.1523/JNEUROSCI. 3644-17.2018.t3-1). This result suggests that GABAergic interneurons other than AMMC-LN and AMMC-B2 also contribute to suppress AMMC-B1 activity. Moreover, whereas the response patterns to varying IPIs were statistically different between AMMC-LN silenced flies and their controls, the difference between AMMC-B2 silenced flies and their controls were not significant (Fig. $5 B-D$; see TNT vs IMPTNT in Table 2 ). The contribution of AMMC-B2 neurons to shape the response pattern of AMMC-B1 neurons might be smaller than that of AMMC-LN neurons.

\section{GABAergic local interneurons suppress the behavioral response to the song}

To elucidate the functional significance of GABAergic local AMMC-LN and AMMC-B2 interneurons on the song response behavior, we investigated whether these interneurons contributed to the female's behavioral response to courtship songs with short IPIs. We set up a behavioral paradigm in which female receptivity for a mute male was monitored upon artificial song playback. Consistent with a previous study (Bennet-Clark and Ewing, 1969), wild-type fly pairs, each of which comprised a virgin female and a wing-clipped virgin male, had increased CR upon playback of pulse songs, especially at their species-specific IPI (i.e., 35 ms; Fig. 6A).

We then observed the CR of genetically manipulated females, in which neurotransmission of the target neurons was silenced by expressing TNT, paired with a wing-clipped wild-type male (see
Table 1 for genotypes; see Fig. 1-1, available at https://doi.org/10. 1523/JNEUROSCI.3644-17.2018.f1-1; Fig. 3-1, available at https:// doi.org/10.1523/JNEUROSCI.3644-17.2018.f3-1; and Fig. 6-1, available at https://doi.org/10.1523/JNEUROSCI.3644-17.2018. f6-1, for labeling patterns of each driver line). First, we silenced the AMMC-B1 neurons to confirm their importance as song relay neurons (Vaughan et al., 2014). Silencing AMMC-B1 neurons in females (TNT group) significantly decreased the accumulated CR during the song playback compared with the accumulated CR of the control females (IMPTNT group; Fig. 6B; Table 4). This result indicates that AMMC-B1 neurons contribute positively to the behavioral response of female flies to the courtship song.

In contrast, silencing either AMMC-LN or AMMC-B2 dramatically increased the CR to short IPIs (Fig. 6C,D; Table 4). When AMMC-LN was silenced, CR increased significantly in all IPI songs tested (Fig. 6C). Conversely, when AMMC-B2 was silenced, CR increased constantly at $15 \mathrm{~ms}$ IPI and partially at 25 and $35 \mathrm{~ms}$ IPIs (Fig. 6D). To compare the suppression levels between different IPIs, we calculated the suppression index (1 $\mathrm{CR}_{\text {IMPTNT }} / \mathrm{CR}_{\mathrm{TNT}}$ ) for each IPI song evoked by either AMMC-LN or AMMC-B2. The song with a $15 \mathrm{~ms}$ IPI yielded the highest suppression index in both AMMC-LN and AMMC-B2 (Fig. 6E). This result reflected the tendency that control flies expressing IMPTNT rarely copulated during a $15 \mathrm{~ms}$ IPI. Together, these results suggest that each local interneuron suppresses the behavioral response to the courtship song, particularly at the $15 \mathrm{~ms}$ IPI song. It should be noted that the accumulated CRs of control females expressing IMPTNT were significantly different from 
A
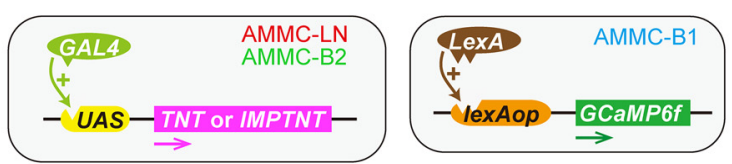

B

\section{AMMC-B1 (AMMC-LN Silenced)}

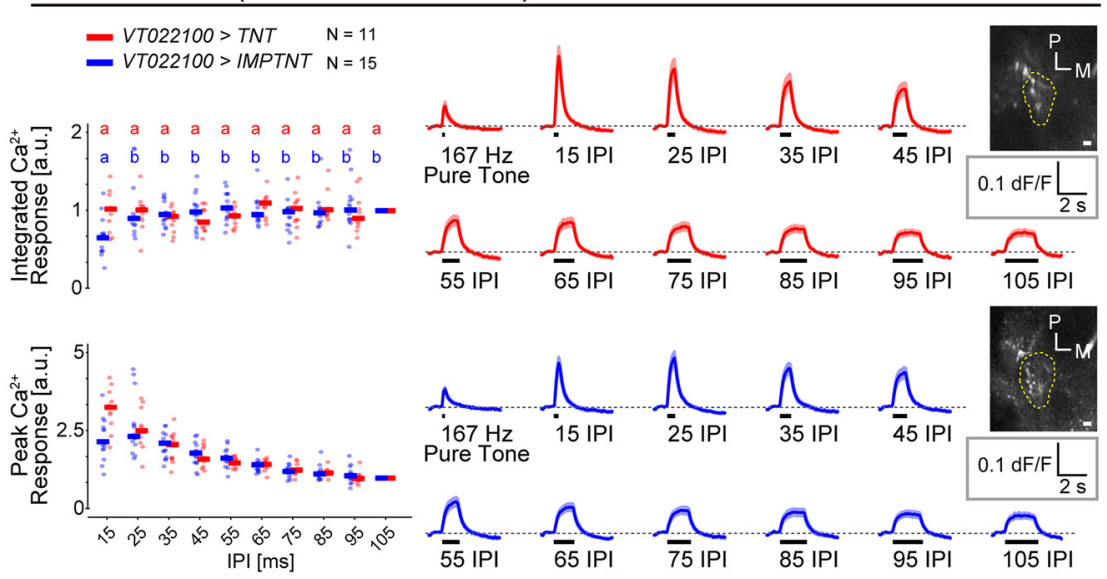

C

AMMC-B1 (AMMC-LN Silenced)

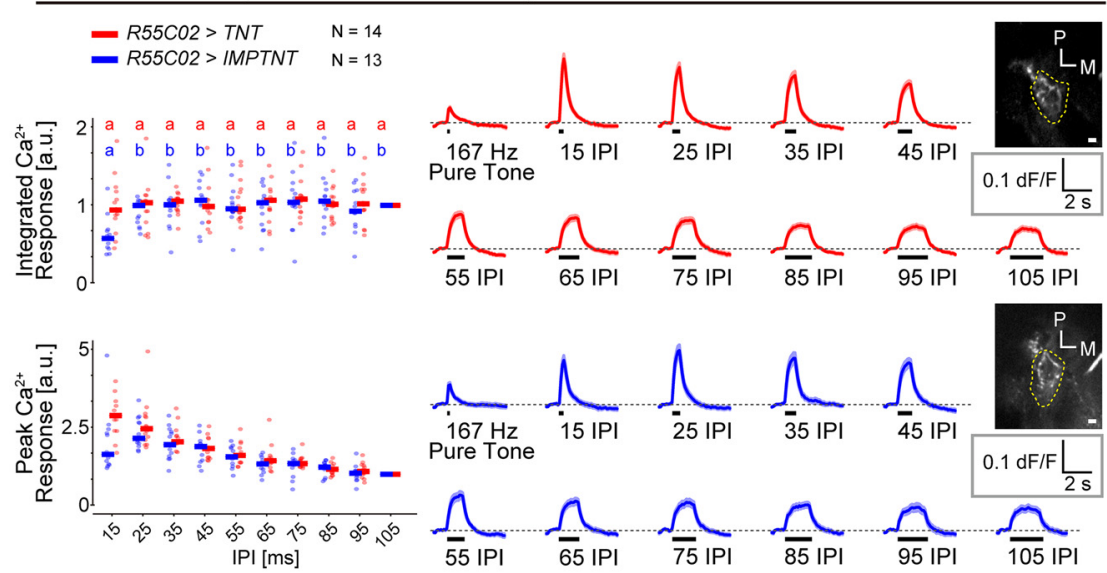

D

AMMC-B1 (AMMC-B2 Silenced)

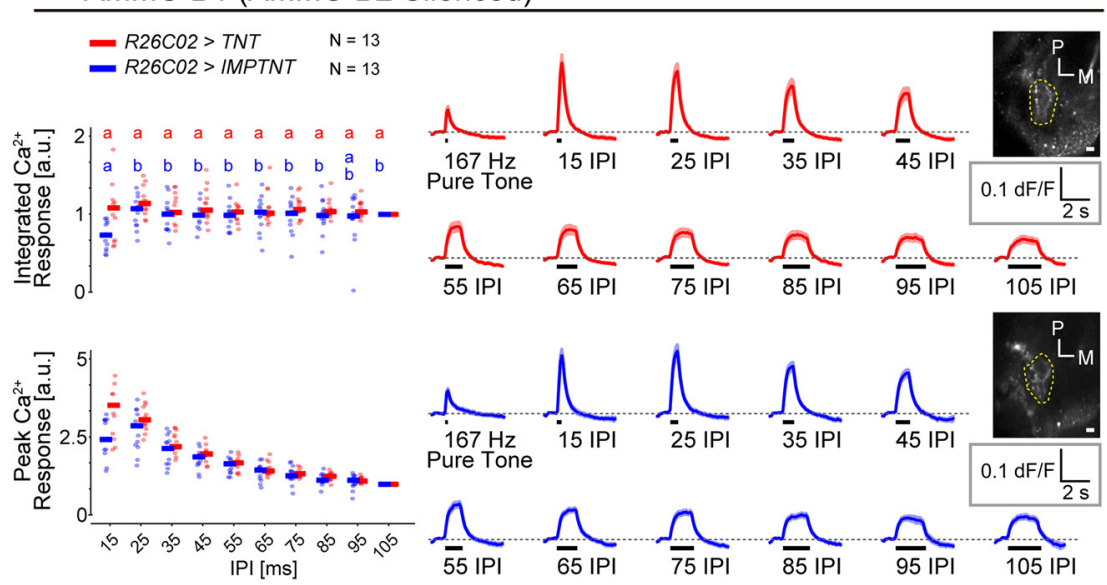

Figure 5. AMMC-B1 neurons receive inhibitory inputs from each local interneuron. $A$, Tools for $\mathrm{Ca}^{2+}$ imaging after neuronal silencing. GAL4/UAS system to express effectors in AMMC-LN or AMMC-B2 neurons is combined with LexA/lexAop system to drive GCaMP6f expression in AMMC-B1 neurons. $\boldsymbol{B}$ - D, AMMC-B1 responses after silencing GABAergic local interneurons. Left, Normalized $\mathrm{Ca}^{2+}$ response of AMMC-B1 neurons when TNT (red) or its inactive form, IMPTNT (blue), was expressed in AMMC-LN (B, $C$ ) or AMMC-B2 (D) neurons by the VT022100 (B), R55C02 (C), or R26C02 (D) GAL4 driver. Right, Time traces of raw $\triangle F / F$ responses. that of wild-type females in most cases (Fig. 6-2, available at https://doi.org/ 10.1523/JNEUROSCI.3644-17.2018.f6-2;

Table 4-1, available at https:/doi.org/ 10.1523/JNEUROSCI.3644-17.2018.t4-1). This difference is presumably due to a difference between GAL4 drivers used, which was also observed in previous studies (Vaughan et al., 2014; Zhou et al., 2014).

\section{Discussion}

In fruit flies, GABA is the major inhibitory neurotransmitter that shapes the neuronal responses via $\mathrm{GABA}_{\mathrm{A}}$ receptors in the early stages of sensory processing, such as in the olfactory and visual systems (Wilson and Laurent, 2005; Freifeld et al., 2013). In the present study, we found GABAergic regulation in the auditory system of female flies; the response pattern of AMMC-B1 neurons, key relay neurons that transmit song information to the mating decision center, was regulated by their $\mathrm{GABA}_{\mathrm{A}}$ receptors. We also suggest that the GABAergic local interneurons AMMC-LN and AMMC-B2 shape the response pattern of AMMC-B1 neurons at a $15 \mathrm{~ms}$ IPI and attenuate the behavioral response to courtship songs in females. Therefore, we propose that AMMC-LN and AMMC-B2 comprise feedforward inhibitory pathways that shape the IPI selectivity of AMMC-B1 neurons and adjust the behavioral response to pulse songs in female flies (Fig. 7).

Local interneurons shape the temporal tuning of a song relay neuron

The present study suggests that the transformation of IPI selectivity from auditory sensory neurons JO-B to AMMC-B1 neurons is the first step of IPI processing in female flies (Fig. 1). As illustrated in Figure 7 , we propose a model in which the IPI selectivity of AMMC-B1 neurons is shaped by the interaction between principal excitatory inputs from JO-B and inhibitory inputs via feedforward pathways containing the GABAergic interneurons AMMC-LN and AMMC-B2.

When we silenced AMMC-LN or AMMC-B2, the AMMC-B1 response to

$\leftarrow$

Responses are displayed as in Figure 1. N, Number of animals tested. Scale bars, $10 \mu \mathrm{m} . M$, Medial; $P$, posterior. Different letters indicate significant differences among IPIs $(\boldsymbol{p}<0.05)(\boldsymbol{B}-\boldsymbol{D}$, left). For the $\mathrm{Ca}^{2+}$ response of AMMC-B1 neurons labeled by $R 45 \mathrm{D07}$ LexA driver, see Figure 5-1A (available at https://doi.org/10.1523/ JNEUROSCI.3644-17.2018.f5-1). For the raw $\Delta F / F$ response of AMMC-B1 neurons when TNT or IMPTNT was expressed in each GABAergic local interneuron, see Figure 5-1B (available at https://doi.org/10.1523/JNEUROS(I.3644-17.2018.f5-1). 
A

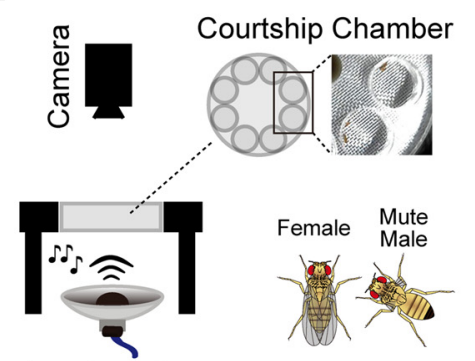

Loudspeaker

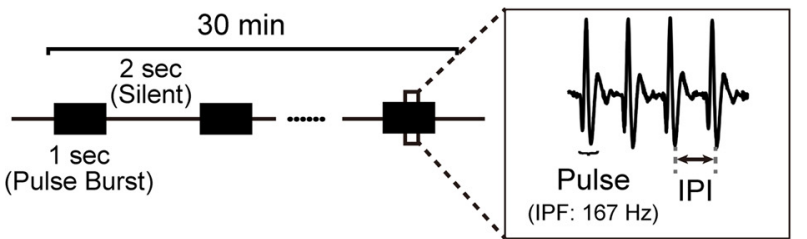

C
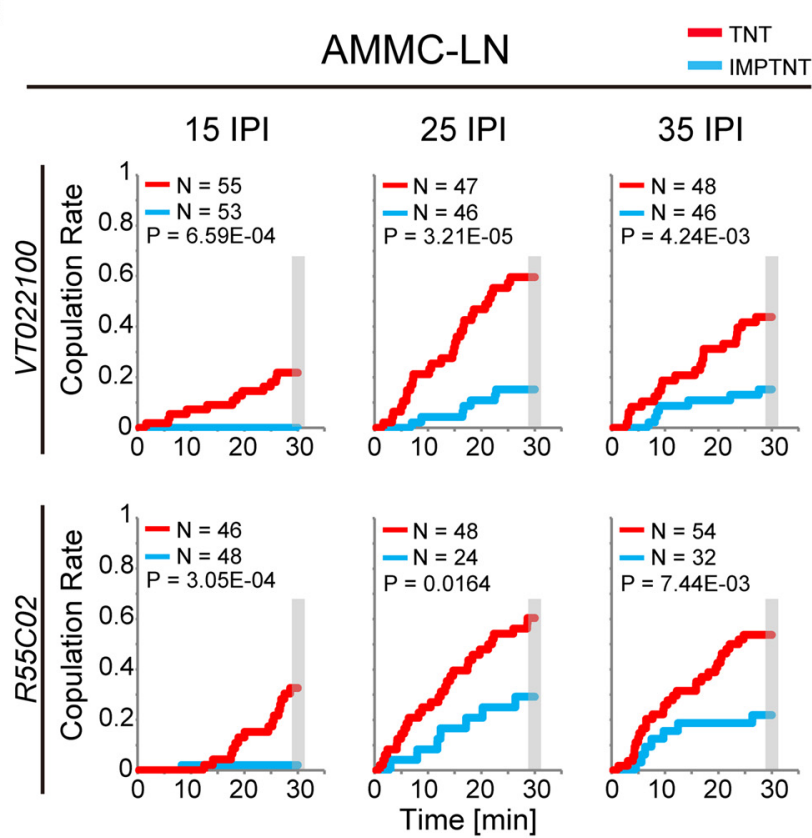

$\mathbf{E}$
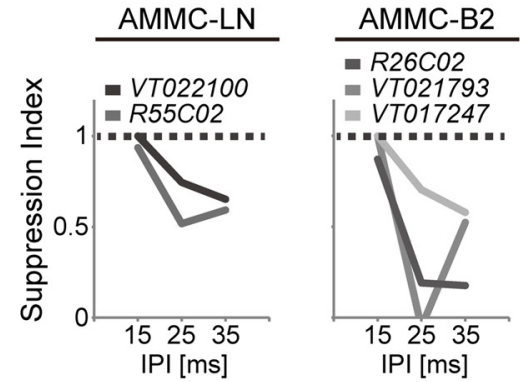

B
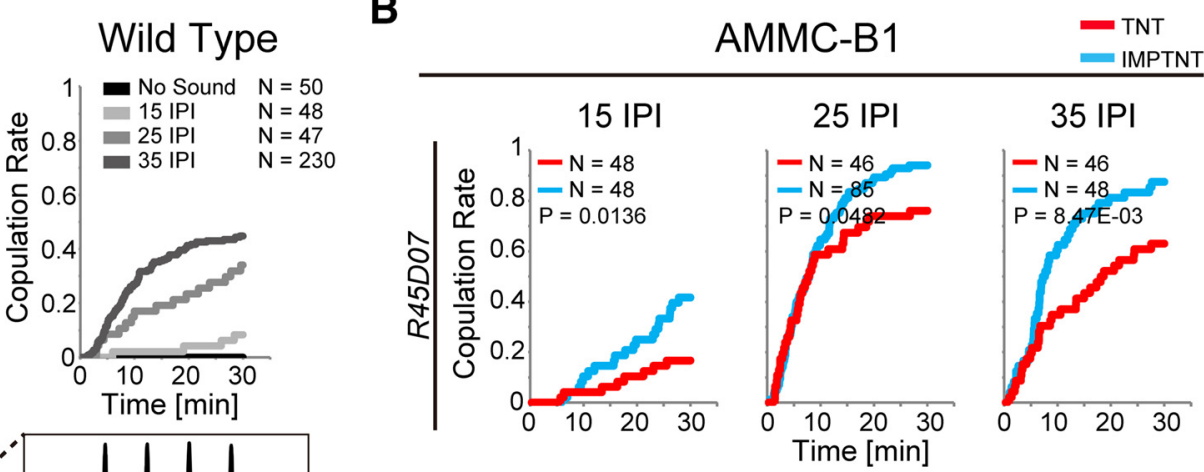

D
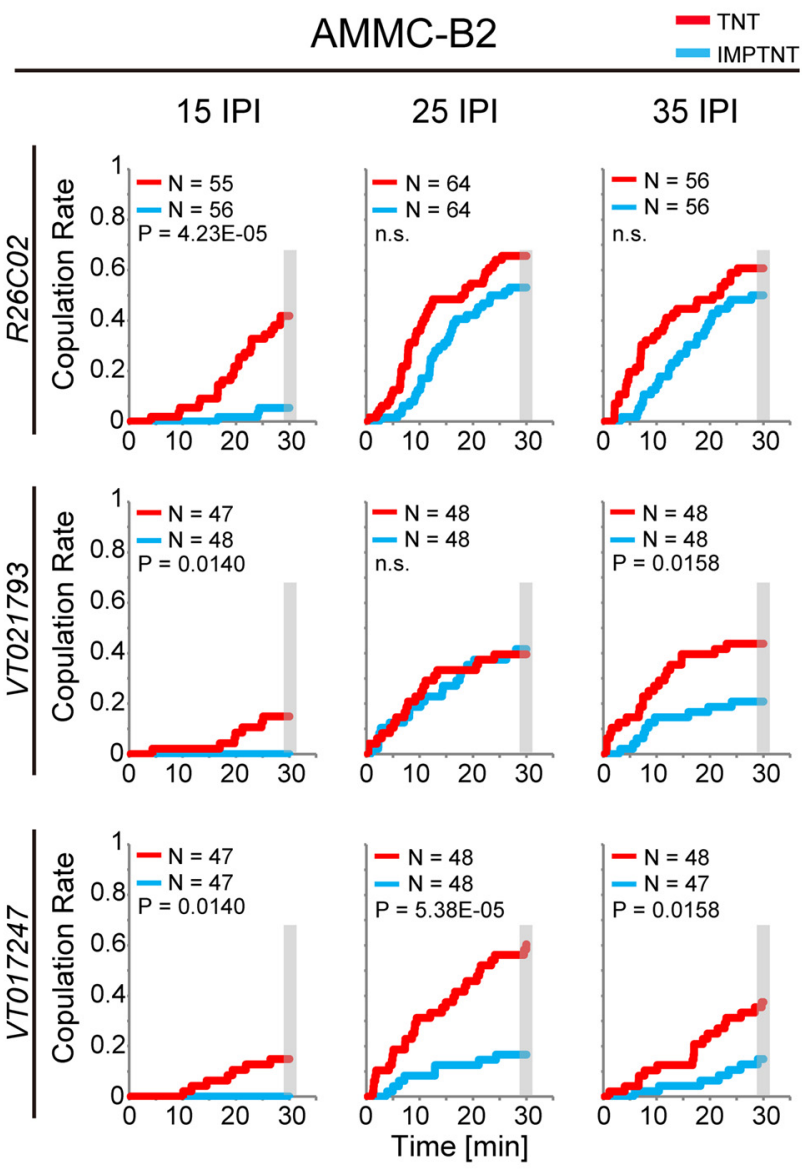

Figure 6. GABAergic local interneurons suppress the female's response to song. $\boldsymbol{A}$, Female receptivity assay. Left, Top, Experimental setup. Bottom, Artificial pulse song. Songs with 15,25 , and $35 \mathrm{~ms}$ IPIs were used. Right, Top, Copulation rate of wild-type fly pairs during playback of artificial pulse songs. Cumulative copulation success rate as a function of time is shown. Inset shows the number of fly pairs. $B$, Copulation rate of females in which AMMC-B1 neurons were silenced. An artificial pulse song was played during the assay. Experimental groups expressed TNT (red), whereas control groups expressed IMPTNT (light blue), in AMMC-B1 neurons of females. Wild-type (Canton-S) male flies were used as partners for the females. The number of fly pairs and $p$-value are shown in each panel. C, Copulation rate of females in which AMMC-LN neurons were silenced. $\boldsymbol{D}$, Copulation rate of females in which AMMC-B2 neurons were silenced. $\boldsymbol{E}$, Suppression index. Behavioral suppression of the copulation rate by each GABAergic local interneuron is plotted as a function of IPI. The copulation rate at 30 min (gray shaded period in $\boldsymbol{C}$ and $\boldsymbol{D}$ ) was used to calculate the suppression index (1 - $\left(\mathrm{R}_{\mathrm{IMPTNT}} / \mathrm{CR}_{\mathrm{TNT}}\right)$. n.S., Not significant. For the labeling pattern of GAL4 driver lines, see Figure 6-1 (available at https://doi.org/10.1523/JNEUROSCI.3644-17.2018.f6-1). For the comparison of copulation rates between control flies, see Figure 6-2 (available at https://doi.org/10.1523/JNEUROSCI.3644-17.2018.f6-2). 
Table 4. Statistical comparison of the copulation assay datasets between silenced and control flies

\begin{tabular}{|c|c|c|c|c|c|c|c|c|}
\hline & Neuronal type & GAL4 line & Sound stimuli & $\chi^{2}$ value & Df & $p$-value & Adjusted $p$-value & Significance \\
\hline \multirow[t]{3}{*}{ Figure $6 B$} & AMMC-B1 & R45D07 & $15 \mathrm{IPI}$ & 6.81 & 1 & $9.04 \mathrm{E}-03$ & 0.0136 & * \\
\hline & & & $25 \mathrm{IPI}$ & 3.90 & 1 & 0.0482 & 0.0482 & $*$ \\
\hline & & & $35 \mathrm{IPI}$ & 8.92 & 1 & $2.82 \mathrm{E}-03$ & $8.47 \mathrm{E}-03$ & $* *$ \\
\hline \multirow[t]{6}{*}{ Figure $6 C$} & AMMC-LN & VT022100 & $15 \mathrm{IPI}$ & 12.9 & 1 & $3.30 \mathrm{E}-04$ & $6.59 \mathrm{E}-04$ & $* * *$ \\
\hline & & & $25 \mathrm{IPI}$ & 20.7 & 1 & 5.35E-06 & $3.21 \mathrm{E}-05$ & $* * *$ \\
\hline & & & $35 \mathrm{IPI}$ & 8.92 & 1 & $2.83 \mathrm{E}-03$ & $4.24 \mathrm{E}-03$ & $* *$ \\
\hline & & R55CO2 & $15 \mathrm{IPI}$ & 15.1 & 1 & $1.02 \mathrm{E}-04$ & $3.05 \mathrm{E}-04$ & $* * *$ \\
\hline & & & $25 \mathrm{IPI}$ & 5.76 & 1 & 0.0164 & 0.0164 & * \\
\hline & & & $35 \mathrm{IPI}$ & 7.49 & 1 & $6.20 \mathrm{E}-03$ & 7.44E-03 & $* *$ \\
\hline \multirow[t]{9}{*}{ Figure $6 D$} & AMMC-B2 & $R 26 \mathrm{CO} 2$ & $15 \mathrm{IPI}$ & 21.0 & 1 & 4.70E-06 & $4.23 \mathrm{E}-05$ & $* * *$ \\
\hline & & & $25 \mathrm{IPI}$ & 3.85 & 1 & 0.0498 & 0.0561 & \\
\hline & & & $35 \mathrm{IPI}$ & 4.20 & 1 & 0.0404 & 0.0519 & \\
\hline & & VT021793 & $15 \mathrm{IPI}$ & 7.65 & 1 & $5.68 \mathrm{E}-03$ & 0.0140 & * \\
\hline & & & $25 \mathrm{IPI}$ & $5.67 \mathrm{E}-03$ & 1 & 0.940 & 0.940 & \\
\hline & & & 35 IPI & 6.66 & 1 & $9.88 \mathrm{E}-03$ & 0.0158 & * \\
\hline & & VT017247 & $15 \mathrm{IPI}$ & 7.49 & 1 & $6.21 \mathrm{E}-03$ & 0.0140 & * \\
\hline & & & $25 \mathrm{IPI}$ & 19.2 & 1 & $1.20 \mathrm{E}-05$ & $5.38 \mathrm{E}-05$ & $* * *$ \\
\hline & & & $35 \mathrm{IPI}$ & 6.54 & 1 & 0.0105 & 0.0158 & $*$ \\
\hline
\end{tabular}

Asterisks indicate statistical significance: ${ }^{* * *} p<0.001,{ }^{* *} p<0.01,{ }^{*} p<0.05$. For a statistical comparison of the copulation assay datasets between control flies, see Table 4-1 (available at https://doi.org/10.1523/JNEUROSCI.3644-17. 2018.t4-1).

Df, degree of freedom.

$15 \mathrm{~ms}$ IPI songs increased significantly (Fig. 5), suggesting that AMMC-LN and AMMC-B2 release GABA, particularly at $15 \mathrm{~ms}$ IPI, to suppress AMMC-B1 activity. The peak $\mathrm{Ca}^{2+}$ responses, more than the integrated $\mathrm{Ca}^{2+}$ responses, of AMMC-LN and AMMC-B2 neurons correlate better with such IPI-dependent transmitter release (Fig. 4), implying a correlation between high instantaneous intracellular $\mathrm{Ca}^{2+}$ concentration $\left(\left[\mathrm{Ca}^{2+}\right]_{\mathrm{i}}\right)$ and the enhanced transmitter release. How is such IPI-dependent transmitter release of AMMC-LN and AMMC-B2 neurons achieved? Experiments using in vivo whole-cell patch clamp revealed that AMMC-B2 neurons exhibited only graded potential responses to sound stimuli (Tootoonian et al., 2012). Because graded potential, or nonspiking, neurons have graded transmitter release (Juusola et al., 1996), synaptic transmission is enhanced as $\left[\mathrm{Ca}^{2+}\right]_{\mathrm{i}}$ increases (Augustine, 2001). We speculate that AMMC-LN also shows graded potential responses to sound stimuli because graded potential neurons are commonly found in insects (Siegler, 1985) and most auditory interneurons in the fruit fly brain show graded potential responses (Clemens et al., 2015). Transmitter release of AMMC$\mathrm{B} 2$, and possibly AMMC-LN, would thus strongly correlate with the IPI dependency of the peak $\mathrm{Ca}^{2+}$ response, which reached its highest level at $15 \mathrm{~ms}$ IPI (Fig. 4). It should be noted that we could not specifically study the presynaptic $\mathrm{Ca}^{2+}$ dynamics in our experiment because our $\mathrm{Ca}^{2+}$ imaging data for AMMC-LN/ AMMC-B2 were acquired from a region
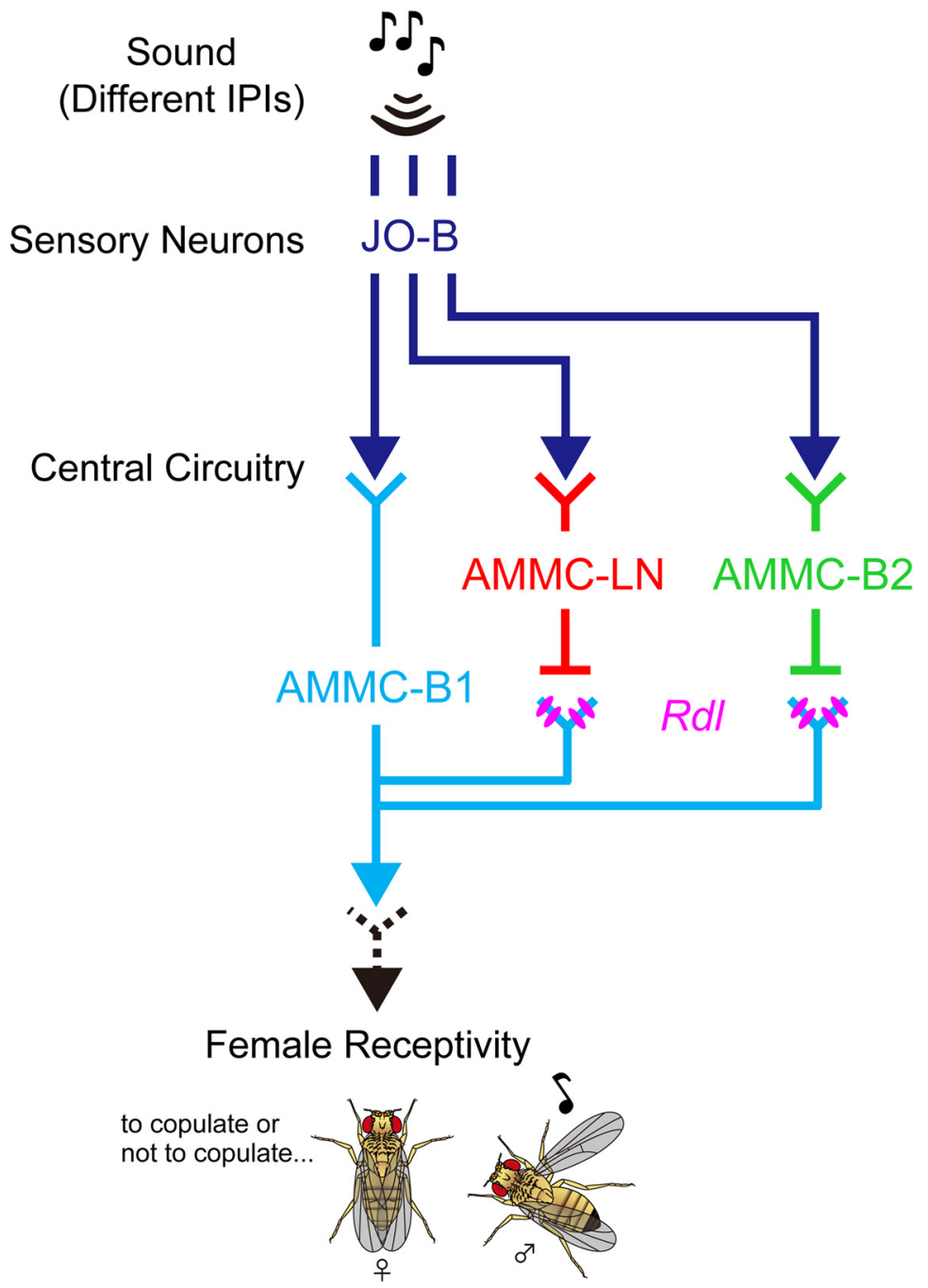

Figure 7. A model of the auditory pathways to relay song signals for controlling female receptivity. Two GABAergic local interneurons (AMMC-LN and AMMC-B2) modulate the response pattern of AMMC-B1 neurons by inhibiting the neuronal activity to the short-IPI song. The dashed line indicates a potential functional connection not explored in this study. 
with intermingled input and output sites. Further analyses are needed to evaluate whether AMMC-LN shows graded potential responses to sound stimuli and to verify the presynaptic $\mathrm{Ca}^{2+}$ dynamics in neurons expressing both presynaptic markers and $\mathrm{Ca}^{2+}$ indicators.

Although we proposed a model for IPI selectivity of AMMC-B1, only the modulation produced by AMMC-LN and AMMC-B2 does not consistently explain the AMMC-B1 response changes observed in our experiments (Rdl knock-down and AMMC-LN/ AMMC-B2 silencing). Rdl knock-down flies had increased $\mathrm{Ca}^{2+}$ responses to IPIs between 15 and $35 \mathrm{~ms}$ (Fig. 2). In contrast, silencing AMMC-LN or AMMC-B2 altered the response pattern at $15 \mathrm{~ms}$ IPI, but not at 25 or $35 \mathrm{~ms}$ IPIs (Fig. 5). These results suggest that GABAergic interneurons other than AMMC-LN and AMMC-

$\mathrm{B} 2$ also contribute to shape the response pattern of AMMC-B1 at short IPIs. Indeed, the dendritic region of AMMC-B1 is distributed more broadly than indicated by the GRASP signals between AMMC-B1 and each local interneuron and also between AMMC-B1 and JO neurons (Lai et al., 2012; Fig. 3). Multiple mechanisms would presumably contribute to the gain control of AMMC-B1, which eventually leads to a strict control of songinduced mating behavior in females. Likewise, in addition to suppressing the AMMC-B1 response at $15 \mathrm{~ms}$ IPI, AMMC-LN and AMMC-B2 are involved in suppressing the behavioral response to the pulse song carrying a broad range of IPIs (Fig. 6). This result suggests that the target of these GABAergic local interneurons is also not confined to AMMC-B1. Broader distribution of the output synapses of both AMMC-LN and AMMC-B2 than that indicated by the GRASP signals between AMMC-B1 and each local interneuron supports this possibility. We previously identified 30 interneurons in the female fly brain that innervate the axonal region of JO-B (Matsuo et al., 2016). Some of them could be the modulation targets of AMMC-LN and AMMC-B2, which together might contribute to the behavioral response to the pulse song as a multilayered neural circuit.

\section{Temporal tuning in flies and other animals}

Our model suggesting that the nature of inhibitory nonspiking neuron generates IPI selectivity is different from previous models. In vertebrates, multiple synaptic mechanisms, temporal summation and/or short-term plasticity of excitatory and inhibitory synaptic inputs, as well as relative timing of these opposing inputs, are suggested to create interval selectivity (Grothe, 1994; Baker et al., 2013; Rose, 2014). These circuit models are, however, based on spiking neurons. In crickets, a delay line coincidence detection circuit comprising four auditory interneurons (LN2-LN5) is suggested, in which the coincidence detector neuron LN3 receives direct and indirect excitatory inputs from an ascending auditory neuron and the feedforward pathway, respectively (Hedwig, 2016). In this pathway, the inhibitory neuron does not have a direct output to LN3, but provides indirect excitation onto LN3 via nonspiking excitatory LN5 with a postinhibitory rebound excitation mechanism. Therefore, although crickets and flies use similar components (i.e., feedforward circuit motif, inhibitory neuron, and nonspiking neuron) to create a circuit for temporal tuning, how they are combined differs in each circuit model.

Even in crickets, the direct causality between the activity of individual neurons and sound-evoked behavior is not well known. The feedforward inhibitory network that we identified and manipulated in the fly brain therefore provides a remarkable example that connects the neural circuit for decoding temporally coded information of the communication sound and its behavioral output.

\section{Feedforward circuit motif to fine-tune mating behavior}

For a fruitful mating of animals, both males and females actively choose an appropriate mating partner by evaluating speciesspecific sensory signals such as visual, olfactory, gustatory, and auditory cues. This decision making is based on information processing of sexual signals with various sensory modalities, so its underlying mechanism is important for understanding the logic of mating decisions. One of the best-studied neural pathways for mating decisions is the neural circuit connecting sensory information to the mating command-like neuron $\mathrm{pC} 1$ in male flies (Clowney et al., 2015; Zhou et al., 2015; Auer and Benton, 2016). In the gustatory pheromone-sensing pathway, both feedforward excitatory and inhibitory circuits transmit sensory information to $\mathrm{pC} 1$ in a balanced manner (Clowney et al., 2015). Together with our proposed feedforward model in the auditory pathway, these common feedforward motifs across modalities might contribute to fine-tune the mating behavior in flies.

In the auditory pathway from the fly ear to $\mathrm{pC} 1$ in males, the neural response to the IPI is shaped gradually at each step so that $\mathrm{pC} 1$ has a band-pass response that peaks between 35 and $65 \mathrm{~ms}$ IPI (Zhou et al., 2015). In females, $\mathrm{pC} 1$ also responds robustly to pulse songs with a $35 \mathrm{~ms}$ IPI and promotes female receptivity (Zhou et al., 2014). Although the response pattern of female pC1 to various IPIs has not been examined, the $\mathrm{Ca}^{2+}$ response of AMMC-B1 neurons, putative upstream neurons of female $\mathrm{pC} 1$, peaks at $25 \mathrm{~ms}$ IPI with a significant reduction in amplitude at shorter IPIs (Vaughan et al., 2014; Fig. 1). This "band-pass" pattern is supportive of species-specific IPI detection. Therefore, although males and females show different motor output in response to the song, a labeled line for transformation of courtship song in males (Zhou et al., 2015) should also exist in females. Because no sexual dimorphisms have been identified in JO neurons and second-order neurons, including AMMC-B1, AMMCLN, and AMMC-B2 (Kamikouchi et al., 2006; Zhou et al., 2015; Matsuo et al., 2016), male and female brains are likely to process song information in a similar way, at least up to the secondary layer of the song relay circuit.

The IPI in the pulse song differs between Drosophila species (Ewing and Bennet-Clark, 1968; Riabinina et al., 2011), suggesting that the multilayered circuit for decoding temporally coded information also exists in other Drosophila species. Further analysis is needed to explore whether this putative neural circuit to prefer their own IPIs in the species-specific courtship song is conserved among Drosophila sister species, which could serve as the neural circuit basis of song preference evolution.

\section{References}

Alder TB, Rose GJ (1998) Long-term temporal integration in the anuran auditory system. Nat Neurosci 1:519-523. CrossRef Medline

Alder TB, Rose GJ (2000) Integration and recovery processes contribute to the temporal selectivity of neurons in the midbrain of the northern leopard frog, Rana pipiens. J Comp Physiol A 186:923-937. CrossRef Medline

Alexander RD (1962) Evolutionary change in cricket acoustical communication. Evolution 16:443-467.

Auer TO, Benton R (2016) Sexual circuitry in Drosophila. Curr Opin Neurobiol 38:18-26. CrossRef Medline

Augustine GJ (2001) How does calcium trigger neurotransmitter release? Curr Opin Neurobiol 11:320-326. CrossRef Medline

Baker CA, Kohashi T, Lyons-Warren AM, Ma X, Carlson BA (2013) Multi- 
plexed temporal coding of electric communication signals in mormyrid fishes. J Exp Biol 216:2365-2379. CrossRef Medline

Benjamini Y, Hochberg Y (1995) Controlling the false discovery rate: a practical and powerful approach to multiple testing. J R Stat Soc Series B 57:289-300.

Bennet-Clark HC, Ewing AW (1969) Pulse interval as a critical parameter in the courtship song of Drosophila melanogaster. Anim Behav 17:755-759. CrossRef

Bradbury JW, Vehrencamp SL (2011) Principles of animal communication, Ed 2. Sunderland, MA: Sinauer Associates.

Brand AH, Perrimon N (1993) Targeted gene expression as a means of altering cell fates and generating dominant phenotypes. Development 118: 401-415. Medline

Chen TW, Wardill TJ, Sun Y, Pulver SR, Renninger SL, Baohan A, Schreiter ER, Kerr RA, Orger MB, Jayaraman V, Looger LL, Svoboda K, Kim DS (2013) Ultrasensitive fluorescent proteins for imaging neuronal activity. Nature 499:295-300. CrossRef Medline

Clemens J, Girardin CC, Coen P, Guan XJ, Dickson BJ, Murthy M (2015) Connecting neural codes with behavior in the auditory system of Drosophila. Neuron 87:1332-1343. CrossRef Medline

Clowney EJ, Iguchi S, Bussell JJ, Scheer E, Ruta V (2015) Multimodal chemosensory circuits controlling male courtship in Drosophila. Neuron 87: 1036-1049. CrossRef Medline

Edwards CJ, Leary CJ, Rose GJ (2007) Counting on inhibition and ratedependent excitation in the auditory system. J Neurosci 27:13384-13392. CrossRef Medline

Estes PS, Ho GL, Narayanan R, Ramaswami M (2000) Synaptic localization and restricted diffusion of a Drosophila neuronal synaptobrevin-green fluorescent protein chimera in vivo. J Neurogenet 13:233-255. CrossRef Medline

Ewing AW, Bennet-Clark HC (1968) The courtship songs of Drosophila. Behaviour 31:288-301. CrossRef

Feinberg EH, Vanhoven MK, Bendesky A, Wang G, Fetter RD, Shen K, Bargmann CI (2008) GFP reconstitution across synaptic partners (GRASP) defines cell contacts and synapses in living nervous systems. Neuron 57: 353-363. CrossRef Medline

Freifeld L, Clark DA, Schnitzer MJ, Horowitz MA, Clandinin TR (2013) GABAergic lateral interactions tune the early stages of visual processing in Drosophila. Neuron 78:1075-1089. CrossRef Medline

Gordon MD, Scott K (2009) Motor control in a Drosophila taste circuit. Neuron 61:373-384. CrossRef Medline

Grothe B (1994) Interaction of excitation and inhibition in processing of pure tone and amplitude-modulated stimuli in the medial superior olive of the mustached bat. J Neurophysiol 71:706-721. CrossRef Medline

Hedwig BG (2016) Sequential filtering processes shape feature detection in crickets: a framework for song pattern recognition. Front Physiol 7:46. CrossRef Medline

Hosie AM, Aronstein K, Sattelle DB, ffrench-Constant RH (1997) Molecular biology of insect neuronal GABA receptors. Trends Neurosci 20:578583. CrossRef Medline

Ishikawa Y, Okamoto N, Nakamura M, Kim H, Kamikouchi A (2017) Anatomic and physiologic heterogeneity of subgroup-A auditory sensory neurons in fruit flies. Front Neural Circuits 11:46. CrossRef Medline

Jefferis GS, Potter CJ, Chan AM, Marin EC, Rohlfing T, Maurer CR Jr, Luo L (2007) Comprehensive maps of Drosophila higher olfactory centers: spatially segregated fruit and pheromone representation. Cell 128:11871203. CrossRef Medline

Juusola M, French AS, Uusitalo RO, Weckström M (1996) Information processing by graded potential transmission through tonically active synapses. Trends Neurosci 19:292-297. CrossRef Medline

Kamikouchi A, Shimada T, Ito K (2006) Comprehensive classification of the auditory sensory projections in the brain of the fruit fly Drosophila melanogaster. J Comp Neurol 499:317-356. CrossRef Medline

Kamikouchi A, Inagaki HK, Effertz T, Hendrich O, Fiala A, Göpfert MC, Ito K (2009) The neural basis of Drosophila gravity-sensing and hearing. Nature 458:165-171. CrossRef Medline

Kim J, Chung YD, Park DY, Choi S, Shin DW, Soh H, Lee HW, Son W, Yim J, Park CS, Kernan MJ, Kim C (2003) A TRPV family ion channel required for hearing in Drosophila. Nature 424:81-84. CrossRef Medline
Kolodziejczyk A, Sun X, Meinertzhagen IA, Nässel DR (2008) Glutamate, GABA and acetylcholine signaling components in the lamina of the Drosophila visual system. PLoS One 3:e2110. CrossRef Medline

Kostarakos K, Hedwig B (2012) Calling song recognition in female crickets: temporal tuning of identified brain neurons matches behavior. J Neurosci 32:9601-9612. CrossRef Medline

Kyriacou CP, Hall JC (1982) The function of courtship song rhythms in Drosophila. Anim Behav 30:794-801. CrossRef

Lai JS, Lo SJ, Dickson BJ, Chiang AS (2012) Auditory circuit in the Drosophila brain. Proc Natl Acad Sci U S A 109:2607-2612. CrossRef Medline

Lai SL, Lee T (2006) Genetic mosaic with dual binary transcriptional systems in Drosophila. Nat Neurosci 9:703-709. CrossRef Medline

Leiss F, Groh C, Butcher NJ, Meinertzhagen IA, Tavosanis G (2009) Synaptic organization in the adult Drosophila mushroom body calyx. J Comp Neurol 517:808-824. CrossRef Medline

Li X, Ishimoto H, Kamikouchi A (2018) Auditory experience controls the maturation of song discrimination and sexual response in Drosophila. eLife 7:e34348. CrossRef Medline

Macpherson LJ, Zaharieva EE, Kearney PJ, Alpert MH, Lin TY, Turan Z, Lee CH, Gallio M (2015) Dynamic labelling of neural connections in multiple colours by trans-synaptic fluorescence complementation. Nat Commun 6:10024. CrossRef Medline

Manning A (1967) The control of sexual receptivity in female Drosophila. Anim Behav 15:239-250. CrossRef Medline

Matsuo E, Yamada D, Ishikawa Y, Asai T, Ishimoto H, Kamikouchi A (2014) Identification of novel vibration-and deflection-sensitive neuronal subgroups in Johnston's organ of the fruit fly. Front Physiol 5:179. CrossRef Medline

Matsuo E, Seki H, Asai T, Morimoto T, Miyakawa H, Ito K, Kamikouchi A (2016) Organization of projection neurons and local neurons of the primary auditory center in the fruit fly Drosophila melanogaster. J Comp Neurol 524:1099-1164. CrossRef Medline

Miyazaki T, Lin TY, Ito K, Lee CH, Stopfer M (2015) A gustatory secondorder neuron that connects sucrose-sensitive primary neurons and a distinct region of the gnathal ganglion in the Drosophila brain. J Neurogenet 29:144-155. CrossRef Medline

Pfeiffer BD, Ngo TT, Hibbard KL, Murphy C, Jenett A, Truman JW, Rubin GM (2010) Refinement of tools for targeted gene expression in Drosophila. Genetics 186:735-755. CrossRef Medline

Pollack GS (2001) Analysis of temporal patterns of communication signals. Curr Opin Neurobiol 11:734-738. CrossRef Medline

Riabinina O, Dai M, Duke T, Albert JT (2011) Active process mediates species-specific tuning of Drosophila ears. Curr Biol 21:658-664. CrossRef Medline

Ritchie MG, Halsey EJ, Gleason JM (1999) Drosophila song as a speciesspecific mating signal and the behavioural importance of kyriacou and hall cycles in D. melanogaster song. Anim Behav 58:649-657. CrossRef Medline

Rose G, Capranica RR (1983) Temporal selectivity in the central auditory system of the leopard frog. Science 219:1087-1089. CrossRef Medline

Rose GJ (2014) Time computations in anuran auditory systems. Front Physiol 5:206. CrossRef Medline

Rose GJ, Leary CJ, Edwards CJ (2011) Interval-counting neurons in the anuran auditory midbrain: factors underlying diversity of interval tuning. J Comp Physiol A Neuroethol Sens Neural Behav Physiol 197:97-108. CrossRef Medline

Schöneich S, Kostarakos K, Hedwig B (2015) An auditory feature detection circuit for sound pattern recognition. Sci Adv 1:e1500325. CrossRef Medline

Siegler MV (1985) Nonspiking interneurons and motor control in insects. Adv Insect Physiol 18:249-304. CrossRef

Sweeney ST, Broadie K, Keane J, Niemann H, O’Kane CJ (1995) Targeted expression of tetanus toxin light chain in Drosophila specifically eliminates synaptic transmission and causes behavioral defects. Neuron 14: 341-351. CrossRef Medline

Tootoonian S, Coen P, Kawai R, Murthy M (2012) Neural representations of courtship song in the Drosophila brain. J Neurosci 32:787-798. CrossRef Medline

Vaughan AG, Zhou C, Manoli DS, Baker BS (2014) Neural pathways for the detection and discrimination of conspecific song in D. melanogaster. Curr Biol 24:1039-1049. CrossRef Medline 
von Schilcher F (1976) The role of auditory stimuli in the courtship of Drosophila melanogaster. Anim Behav 24:18-26. CrossRef

Wang JW, Wong AM, Flores J, Vosshall LB, Axel R (2003) Two-photon calcium imaging reveals an odor-evoked map of activity in the fly brain. Cell 112:271-282. CrossRef Medline

Wang J, Ma X, Yang JS, Zheng X, Zugates CT, Lee CH, Lee T (2004) Transmembrane/juxtamembrane domain-dependent dscam distribution and function during mushroom body neuronal morphogenesis. Neuron 43: 663-672. CrossRef Medline

Wilson RI, Laurent G (2005) Role of GABAergic inhibition in shaping odorevoked spatiotemporal patterns in the Drosophila antennal lobe. J Neurosci 25:9069-9079. CrossRef Medline
Yan J, Suga N (1996) The midbrain creates and the thalamus sharpens echodelay tuning for the cortical representation of target-distance information in the mustached bat. Hear Res 93:102-110. CrossRef Medline

Yuan Q, Song Y, Yang CH, Jan LY, Jan YN (2014) Female contact modulates male aggression via a sexually dimorphic GABAergic circuit in Drosophila. Nat Neurosci 17:81-88. CrossRef Medline

Zhou C, Pan Y, Robinett CC, Meissner GW, Baker BS (2014) Central brain neurons expressing doublesex regulate female receptivity in Drosophila. Neuron 83:149-163. CrossRef Medline

Zhou C, Franconville R, Vaughan AG, Robinett CC, Jayaraman V, Baker BS (2015) Central neural circuitry mediating courtship song perception in male Drosophila. eLife 4:e08477. CrossRef Medline 